University of South Carolina

Scholar Commons

\title{
$5-2014$
}

\section{The Impact of Mindfulness on Emotion Dysregulation and Psychophysiological Reactivity under Emotional Provocation}

Tanya S. Watford

University of South Carolina - Aiken

Follow this and additional works at: https://scholarcommons.sc.edu/aiken_psychology_theses

Part of the Psychology Commons

\section{Recommended Citation}

Watford, Tanya S., "The Impact of Mindfulness on Emotion Dysregulation and Psychophysiological Reactivity under Emotional Provocation" (2014). USC Aiken Psychology Theses. 6.

https://scholarcommons.sc.edu/aiken_psychology_theses/6

This Thesis is brought to you by the Psychology Department at Scholar Commons. It has been accepted for inclusion in USC Aiken Psychology Theses by an authorized administrator of Scholar Commons. For more information, please contact digres@mailbox.sc.edu. 
The Impact of Mindfulness on Emotion Dysregulation and Psychophysiological Reactivity under Emotional Provocation

A Thesis
Presented to
The Faculty of the Department of Psychology
University of South Carolina Aiken

In Partial Fulfillment

Of the Requirements for the Degree

Master of Science

By

Tanya S. Watford

May 2014 


\begin{abstract}
The present study employed physiological measures and a working memory task in addition to self-report measures to seek a better understanding of the relationship between brief mindfulness training and the experience and regulation of emotion. Seventy undergraduate students at a small southern state university completed baseline measures of trait mindfulness and emotion regulation before experiencing a 15-minute recording (mindfulness or control), and then completing a state mindfulness measure. Participants then experienced an emotion induction (positive or negative), before completing state emotion dysregulation and affect measures, and then completing a working memory task, finishing with the state mindfulness measure again. Physiological measures were recorded throughout the experimental session. Results indicated that the mindfulness induction was sufficient to increase mindfulness, demonstrated by greater self-report of state mindfulness, greater L > R frontal brain asymmetry, and greater heart rate variability at the completion of the intervention as compared to the Control group. Further, participants receiving the mindfulness induction experienced greater emotional awareness, indicated by reporting greater positive affect regardless of induction and greater negative affect when experiencing a negative induction. Experiencing a negative emotion induction after mindfulness training also resulted in feeling more overwhelmed and unable to improve their emotional state, suggesting the mindfulness induction was successful in reducing emotional avoidance, but failed to improve emotion regulation capacity sufficiently to withstand the demands of an aversive emotional experience. These results have significant clinical implications since it appears that individuals may feel more dysregulated while initially experiencing increased mindfulness.
\end{abstract}


The Impact of Mindfulness on Emotion Dysregulation and Psychophysiological Reactivity under Emotional Provocation

Mindfulness is a mental state characterized by particular qualities of attention and awareness that has its origins in Buddhist and other Eastern meditative traditions. Jon KabatZinn (2003) offered the following definition, stating that mindfulness is: "the awareness that emerges through paying attention on purpose, in the present moment, and nonjudgmentally to the unfolding of experience moment by moment" (p. 145). Mindfulness is the central aspect of Buddhist meditative practice, aimed at methodically training and cultivating various aspects of the mind through the use of mindful attention, and the Buddhist tradition may be credited with refining and articulating this simple and effective practice (Kabat-Zinn, 2003). However, attention is an inherent human capacity, and as such we are all mindful to some degree, making mindfulness universal (Kabat-Zinn, 2003).

In the last 40 years, the practice of mindfulness has been incorporated into psychological research and practice (Batchelor, 1994). Mindfulness and acceptance-based interventions, such as Dialectical Behavior Therapy (DBT; Linehan, 1993), Mindfulness-Based Stress Reduction (MBSR; Kabat-Zinn, 1990), and Acceptance and Commitment Therapy (ACT; Hayes, Strosahl, \& Wilson, 1999), have been evaluated and found to be effective treatment for a wide range of psychological difficulties (Baer, 2003). Research has indicated the benefits of mindfulness training for preventing the relapse of depression (Teasdale et al.,2000), reducing anxiety and negative affect (Shapiro, Brown, \& Biegel, 2007), reducing pain sensations (Perlman, Salomons, Davidson, \& Lutz, 2010), reducing negative automatic thoughts (Frewen, Evans, Maraj, Dozois, \& Partridge, 2007), promoting brain activity in areas associated with positive emotion (Davidson et al, 2003), and improving working memory (van Vugt, \& Jha, 2011). Despite the initial support 
of mindfulness and its associated treatments, several researchers (e.g., Davidson, 2010; Lynch, Chapman, Rosenthal, Kuo, \& Linehan, 2006) have suggested more research is needed to establish the mechanisms through which mindfulness increases psychological functioning and decreases psychological distress.

Furthermore, in the past the study of mindfulness has often relied solely on self-report measures like the Kentucky Inventory of Mindfulness Skills (KIMS; Baer et al., 2004), and emotional ratings using a Likert scale (e.g., Hill \& Updegraff, 2012). Despite the strength of reliability and validity of many of the measures utilized in this research, the sole use of subjective measures leaves many questions unanswered, and is often cited as a limitation in this literature (e.g., Vujanovic et al., 2010). Thus, studies that employ more objective measures of the effects of mindfulness provide a more accurate understanding of the mechanisms of action. Several investigators, including Vujanovic et al. (2010) and Erisman and Roemer (2010), have recommended the use of physiological measures and performance on cognitive tasks to further explore the mechanisms of change engendered by mindfulness practice.

\section{Mindfulness and Emotion Regulation}

Previous research has demonstrated a significant relationship between mindfulness and a construct termed emotion regulation (e.g., Hill \& Updegraff, 2012). Emotion regulation has been defined as the ability to reduce or control negative emotions (Erisman \& Roemer, 2010). Other definitions include the ability to move toward valued goals in spite of difficult emotions (Hayes \& Feldman, 2004) and also as the process of amplifying, attenuating, or maintaining the strength of emotional reactions (e.g., subjective experience, expressive behavior, and physiological responses; Davidson, 2000). Emotion dysregulation (i.e., difficulties with emotion regulation) has been associated with a variety of forms of psychopathology, including anxiety (Olatunji, 
Forsyth, \& Feldner, 2007), depression (Ehring et al., 2010), anger and aggression (Pond, Kashdan, DeWall, Savostyanova, Lambert, \& Fincham, 2012) and decline in interpersonal relationship quality (Smith et al., 2011). As such, it is important to continue to explore ways to improve emotion regulation, and to decrease emotion dysregulation. It may be that mindfulness has been effective in reducing human suffering by improving an individual's ability to engage in successful emotion regulation (e.g., Shapiro et al., 2007; Davidson et al., 2003).

The down-regulation of emotion is a common and valued emotion regulatory goal and efforts to down-regulate emotion vary in form and effectiveness. This includes response-focused emotion regulation which entails attempts to suppress an emotion that has already occurred, and antecedent-focused emotion regulation which entails the reappraisal of emotional stimuli in neutral terms (Gross \& Thompson, 2007). Gross (2002) demonstrated that attempts to suppress emotion minimize the external expression of emotion, but have little to no effect on the private, internal experience of emotion, and may become problematic. Suppression, a response- focused form of emotional avoidance, has been cited by Hayes and Feldman (2004) as being associated with worse psychological outcomes. In contrast, these authors suggest antecedent-focused forms of emotion regulation, like neutral reappraisals of an emotional event, are more effective in the down-regulation of the internal, private experience of emotion and result in greater well-being. For example, a study by Ehring et al. (2010), which asked participants to employ either emotion suppression or emotion reappraisal while viewing sad film clips, found that reappraising emotions in neutral terms led to the expression and experience of less negative emotion. Specifically, in the suppression condition participants were instructed, "If you have any feelings as you watch the film, please try your best not to let those feelings show. In other words, as you watch the film clip, try to behave in such a way that a person watching you would not know that 
you were feeling anything. Watch the film clip carefully, but please remember not to let your feelings show." In the reappraisal condition participants were instructed, "Please try to adopt a neutral and unemotional attitude as you watch the film. In other words, as you watch the film, try to concentrate on what you are seeing objectively. Imagine that you are a director and watch the film in terms of the technical aspects of the film, how certain moods are produced, and what cuts and camera angles are used. Watch the film clip carefully, but please remember to think about what you are seeing in such a way that you don't feel anything at all." With participants' scores on the PANAS Negative Mood scale as the dependent variable, analyses showed that participants in the reappraisal condition experienced significantly less negative mood during the film than those in the emotion suppression condition. The results of these studies support the assertion by Hayes and Feldman (2004) that avoidance of negative experiences, which includes the strategy of suppression, is associated with worse psychological outcomes and that forms of emotion regulation that are more effective in the down-regulation of the internal, private experience of emotion (in this instance, reappraisal) result in a greater sense of well-being.

Mindfulness has been posited by several researchers to influence emotion regulation through the facilitation of a more adaptive relationship with one's emotions. Lynch et al. (2006) suggested that mindfulness may change the automatic response tendencies by changing the behavioral response to emotions (i.e., external expression) as well as associated thoughts, memories and images (i.e., internal experiences). Lynch and her colleagues (2006) elaborated further, stating that it seems when a person observes, describes and participates in the experience of their emotions without attempts to alter them or act on them in any way, the meaning of the event (i.e., "it is bad") is altered automatically to "it just is", reducing secondary emotional, cognitive, or behavioral responses that would typically lead to greater suffering. Hayes and 
Feldman (2004) also suggest that mindfulness may act upon the human tendencies to avoid and to over-engage with internal experiences, representing an emotional balance that involves acceptance of these experiences, affective clarity and an ability to regulate one's emotions and moods. This would suggest that mindfulness represents a balance between avoidance and overengagement that would increase cognitive flexibility and well-being. Emotion regulation as a potential mechanism is described by Shapiro et al. (2006) in terms similar to Lynch and colleagues, stating that mindfulness interrupts automatic, maladaptive habits, taking control away from emotions and thoughts as they arise, and eliminating the habitual reactive pattern that would follow. They suggested that the ability fostered by mindfulness to stand back from thoughts and feelings and to observe them creates freedom from them and access to them for information. Therefore, to bring acceptance and awareness to experiences in the present moment through mindfulness, one is able to attend to emotion and engage in self-regulation that facilitates well-being (Shapiro et al., 2006) and psychological flexibility (i.e., the ability to respond adaptively to mental processes that contribute to emotional distress and maladaptive behavior).

The theory that mindfulness facilitates a more adaptive relationship with one's emotions has been explored by several lines of research, including the study of a college population by Vujanovic, Bonn-Miller, Bernstein, McKee and Zvolensky (2010). They found that participants with higher self-report of mindfulness skills also reported less emotion dysregulation. Specifically, they found that accepting without judgment, a mindfulness skill assessed by the Kentucky Inventory of Mindfulness Skills (KIMS; Baer et al., 2004), was negatively correlated with all facets of emotion dysregulation as measured by the Difficulties in Emotion Regulation Scale (DERS; Gratz \& Roemer, 2004). The DERS assesses six facets of difficulty in regulating 
emotion: lack of emotional awareness, lack of emotional clarity, nonacceptance of emotional responses, lack of goal-directed behavior, impulsivity, and limited access to emotion regulation strategies.

Hill and Updegraff (2012) examined the relationship between mindfulness and aspects of emotion regulation, including emotion differentiation, emotion lability (or shifting between emotions) and emotional difficulties, by employing experience sampling in college undergraduates. In addition to completing self-report measures of mindfulness and emotion regulation, participants reported at predetermined intervals throughout the day their current subjective emotions on a Palm Pilot by rating 21 emotions on a Likert scale. Emotions varied on the dimension of pleasantness-unpleasantness (e.g., positive emotions: happy, content, peaceful; negative emotions: sad, ashamed, nervous) and participants rated their experience of these emotions 1 (not at all) to 7 (a great deal). Hill and Updegraff (2012) considered higher correlations between the Palm Pilot ratings of similarly valenced emotions to reflect lower differentiation; thus, they defined emotion differentiation as the ability to distinguish between similarly valenced emotions (i.e., ratings of the individual's experience of similarly valenced emotions, such as "happy" and "content", would differ). The researchers found that emotion differentiation mediated the relationship between mindfulness and emotion lability. In other words, participants reporting greater mindfulness also displayed greater ability to differentiate between the experienced emotions (i.e., lower correlations between similarly valenced emotions) which in turn was associated with greater stability in their emotional state (i.e., less emotion lability). In addition, emotion regulation (as defined by the six facets of the DERS) mediated the relationship between mindfulness and both negative emotion lability and positive emotion differentiation. This finding is particularly relevant to the current study since it means that 
participants reporting greater mindfulness also reported less emotion dysregulation, which demonstrated a direct relationship with reduced reactivity to negative emotional experiences and greater present moment awareness of positive emotional experiences. The main finding of this experience sampling study showed self-reported levels of mindfulness to be related to higher levels of emotional differentiation, providing further support for the association between mindfulness and emotional awareness in the present moment (Hill \& Updegraff, 2012). Furthermore, this supports the assertion by Erisman and Roemer (2010) that emotional awareness is an important aspect of emotion regulation, and that mindfulness may improve emotion regulation by increasing awareness.

Employing mindfulness to bring acceptance and awareness to emotional experiences in the present moment allows one to attend to and engage in a more adaptive relationship with one's emotions (Hayes \& Feldman, 2004). Given that it appears that mindfulness improves emotion regulation (e.g., Vujanovic et al., 2010) and that emotion regulation is related to increased psychological flexibility (e.g., Ehring et al., 2010), it appears that emotion regulation may mediate the relationship between mindfulness and improved psychological functioning. Mindfulness, which brings attention and nonjudgmental awareness to emotion, may be considered the opposite of emotional avoidance, which Hayes and Feldman (2004) theorize is associated with negative psychological outcomes. Therefore, by employing mindfulness one is able to attend to emotion instead of employing avoidance and to respond adaptively (i.e., successful emotion regulation) to mental processes that would otherwise result in emotional distress and maladaptive behavior. Several investigators have suggested this and research examining this mediational model would further elucidate the mechanism of action of mindfulness (e.g., Shapiro et al., 2007; Davidson et al., 2003; Jha et al., 2010). 


\section{Mindfulness Training}

Mindfulness training is a critical aspect of research exploring the effects of mindfulness and researchers have employed various forms of mindfulness training, from long (e.g., a one month retreat; van Vugt \& Jha, 2011) to brief (e.g., 10 minutes during the experimental manipulation; Erisman \& Roemer, 2010). Training may be facilitated through meditation CDs for practice at home or may be delivered in an academic or clinical setting by a trained practitioner. Mindfulness training, no matter its duration or location, aims to enhance one's attention to the present moment and nonjudgmental acceptance of experience (i.e., thoughts, emotions, memories and physical sensations), often through the use of multiple mindfulness techniques.

Goldin and Gross (2010) examined the effects of Mindfulness-Based Stress Reduction (MBSR) on emotion regulation using a pre- and post-training design. MBSR, the most studied form of mindfulness training in the United States (Kabat-Zinn, 1990), consists of eight weekly small group sessions lasting about 2 hours each and includes formal and informal meditation practices. Examples of formal practices include breath-focused attention, body scan-based attention to sensory experience, and open monitoring of present moment experiences without explicit focus on any one thing. Taking a brief pause to shift one's attention to awareness of the

present moment is an example of informal practice. Sears and Kraus (2009) also used a pre- and post- training design to examine the effects of mindfulness meditation on anxiety, positive and negative affect, and hope. Their mindfulness training consisted of 12 weekly guided meditation sessions lasting about 10-15 minutes each wherein participants received either a brief mindful attention or brief loving kindness-focused meditation training. The mindfulness training in these 
examples occurred over many weeks, although the former's sessions lasted 2 hours and the latter's lasted only $10-15$ minutes.

However, if the researcher is interested in examining the effects of mindfulness within the time frame of a single experimental session, mindfulness training such as the brief one employed by Erisman and Roemer (2010) is more appropriate. Erisman and Roemer (2010) employed a brief mindfulness training which consisted of a recorded presentation of the rationale for practicing mindfulness, an experiential exercise in breath-focused attention, presentation of the rationale for applying mindfulness to emotional experiences, and another experiential exercise applying mindfulness to emotional experiences. While some research explains that mindfulness is a skill that can be improved with practice (e.g., Baer et al., 2003), the brief training of Erisman and Roemer (2010) was shown to be effective in producing increased mindfulness in an experimental condition. Participants in both the mindfulness and the control condition completed the Toronto Mindfulness Scale (TMS) both immediately following training as well as at the completion of the experimental session (approximately an hour after the training). Those in the mindfulness condition reported, on average, higher levels of decentering, a measure of state mindfulness, than those in the control condition, suggesting that this brief tenminute training is effective in increasing mindfulness.

\section{The Effect of Mindfulness Training on Emotion Regulation}

Some experimental studies of the effects of mindfulness on emotion regulation have examined behavioral as well as neural measures of emotional reactivity and regulation. In one study, Goldin and Gross (2010) employed a negative self-belief task and fMRI to investigate changes in emotion reactivity to negative self-beliefs due to mindfulness training. Participants asked "How negative do you feel right now?" provided an emotional rating from 1 (Not at all) to 
5 (Very) in response to 18 self-critical personal beliefs (e.g., "People always judge me") while receiving fMRI scanning both before and after an 8-week training in Mindfulness-Based Stress Reduction. During the negative emotion rating task administered post-training, a prompt was included to employ attention regulation, either breath-focused attention (a mindfulness skill learned in training) or distraction-focused attention (non-mindful attention by counting backward from 168) to determine if changes in negative experience were due exclusively to mindful attention. Employing the breath-focused attention resulted in a decrease, from base-line to posttraining, in reported negative experience, reduced amygdala activity (indicating less emotional reactivity) and increased activity in brain areas related to attention. There were no significant differences when participants employed distraction-focused attention between baseline and posttraining measures (Goldin \& Gross, 2010). These results suggest that mindfulness skills specifically may facilitate the ability to attenuate avoidance and implement attentional deployment, increasing adaptability and reducing reactivity in the context of negative self-related emotions.

Farb, Anderson, Mayberg, Bean, McKeon, and Segal (2010) employed fMRI to examine mindfulness training and its relationship to the neural and behavioral expression of sadness. Participants were randomly assigned to either a wait-listed control group or mindfulness training (MT) group, which received 8 weeks of mindfulness training. After the MT group completed training, all participants underwent sadness provocation and provided a rating of their level of sadness after watching sad and neutral film clips while receiving fMRI scans. The results of this study indicate that the MT group demonstrated reduced reactivity in the areas associated with self-referential processing and greater activation in areas associated with interoceptive awareness. The neural patterns in this study suggest that mindfulness may reduce emotional 
interference through the detached viewing of emotion as innocuous sensory information.

Furthermore, the neural patterns suggest that mindfulness increases present moment awareness of sensations in the body (indicated by increased activation of sensory visceral areas of the brain) which may reduce the interpretation of emotional experience as affect-laden and threatening. In this way, mindfulness may support a balance between affective and sensory neural networks (Farb et al., 2010), which promotes emotional awareness and nonjudgmental acceptance. Finally, while the MT group and the control group had similar levels of self-reported sadness, the MT group demonstrated less neural reactivity to the sadness provocation. Despite the lack of significant differences demonstrated by participants self-report, the more objective neural measure still facilitated the elucidation of the effects of mindfulness training. Therefore, the results of this study provide an example of the value of non-self-report measures in examining the effects of mindfulness training.

\section{Brain Asymmetry as a Measure of Emotion Experience and Regulation}

Researchers have assembled a variety of evidence employing electrophysiology (i.e., electroencephalogram, or EEG) suggesting that the two hemispheres of the brain, specifically the frontal and anterior temporal regions, respond differentially to positive and negative emotions (e.g., Davidson et al., 1990; Tomarken, Davidson, \& Henriques, 1990), and this differential activation is known as frontal brain asymmetry. According to this evidence, greater activation of the left anterior brain regions indicates the experience or expression of positive emotions (e.g., happiness) and greater activation of right anterior brain regions indicates the expression or experience of negative emotions (e.g., fear or disgust). For example, Davidson et al. (1990) cued participants to make positive or negative facial expression while recording frontal brain asymmetry and found evidence that greater relative activation of the right anterior frontal lobe 
indicated the experience of negative emotions (i.e., frowning) and that greater relative left activation indicated the experience of positive emotions (i.e., smiling). These results suggest that EEG asymmetry is associated with discrete emotional experience.

There is also evidence to indicate that a differential activation of the frontal and anterior temporal regions of the brain prior to emotional stimulation, known as resting brain asymmetry (Davidson \& Fox, 1989), is related to a flexible pattern of affective responding (e.g., Tomarken et al., 1990; Papousek, Reiser, Weber, Freudenthaler \& Schulter, 2012). In other words, frontal brain asymmetry at rest is positively correlated with an individual's ability to engage in a more adaptive relationship with their emotions, and is a reliable measure of trait emotion regulation. For example, Davidson and Fox (1989) examined the relationship between temperament, defined as individual differences in the experience and expression of emotion, and frontal brain asymmetry by observing the behavior of infants when separated from their mothers. Infant response to this stressor (i.e., separation from their mother) has been associated with individual differences in vulnerability to distress (Davidson \& Fox, 1989). They found that infants exhibiting greater resting right frontal activation were likely to exhibit distress (i.e., cry) when separated from their mothers, as opposed to infants with greater left frontal activation at rest who were not likely to cry. These results suggest that a Left $>$ Right activation at rest is related to a predisposition for adaptive emotional responding. Papousek et al. (2012) examined the relationship of resting frontal brain asymmetry and affective flexibility, which refers to not only emotional responsivity to an affective challenge, but also to emotion regulation and recovery after the offset of the challenge. Findings of this study also supported the relationship between frontal brain asymmetry and the experience of discrete emotions. Following a rest period, participants listened to sound clips through headphones that alternated neutral-sad-neutral- 
cheerful-neutral with instructions to keep their eyes closed and give their full attention to the sounds (Emotionally Contagious Sound Clips; Weber, Papousek, \& Schulter, 2011). No regulatory instructions, such as reappraising emotions, were given, and participants were asked to provide an affect rating after each sound clip. Participants with Right $>$ Left activation at rest demonstrated no significant change in activation during the presentation of the sound clips, indicating that these individuals were limited in their ability to respond affectively. This resting pattern frequently results in emotional response patterns characteristic of depression (Papousek et al., 2012). However, a Left $>$ Right activation pattern in the frontal brain at rest was associated with a shift to Right $>$ Left activation during negative sound clips and a shift to Left $>$ Right activation during positive film clips, as well as efficient recovery of the Left $>$ Right activation after negative stimulation. There is evidence that this kind of flexible emotional responding and efficient recovery associated with Left $>$ Right prefrontal activation at rest indicates the ability to regulate emotional responses (Ochsner, Bunge, Gross, \& Gabrieli, 2002). Furthermore, the findings that a shift to Right $>$ Left activation occurred during negative sound clips and a shift to Left $>$ Right activation occurred during positive film clips support the theory that frontal brain asymmetry is a robust measure of the private experience of discrete emotions. In summary, it appears that frontal brain asymmetry is both a measure of affective response to an emotional experience and also a trait measure of ability to respond adaptively to emotional experience.

\section{Mindfulness, Working Memory Capacity and Emotion Regulation}

Another objective measure of cognitive activity that appears to have relevance to emotion regulation is working memory. Working memory refers to the capacity to maintain information while simultaneously engaging in other cognitive operations, and is often measured by tasks such as the operation span task (OSPAN). OSPAN requires participants to solve a series of math 
problems while simultaneously trying to remember a sequence of unrelated letters, ranging from three to seven in length (Jha et al., 2010). Several lines of research have asserted that working memory capacity (WMC) makes it possible for an individual to maintain goal-relevant processing despite contending response tendencies, which means individual's with higher WMC performed better on the Stroop color-word interference task (Kane \& Engle, 2003), had greater control of visual attention (Kane, Bleckley, Conway, \& Engle, 2001), and successfully ignored unimportant cues (Conway, Cowan, \& Bunting, 2001). Schmeichel et al. (2008) examined the likelihood that individual's with greater WMC would be more successful at emotion regulation. Participants performed working memory tasks (including the OSPAN) and then viewed film clips (i.e., depicting mutilated animals) to induce negative emotion, with instructions to either view the clips as they normally would at home (natural condition), or to adopt a detached, unemotional attitude (neutral-appraisal condition). Participants in the neutral-appraisal condition with higher WMC (i.e., one standard deviation or greater above the mean task reaction time) experienced less negative affect than those in the neutral-appraisal condition with lower WMC (i.e., one standard deviation or lower below the mean task reaction time). WMC was not related to emotional expression or experience in the non-regulatory condition (natural condition). These findings suggest that working memory capacity facilitates the ability to adopt emotion regulation strategies and that participants with lower WMC compared to those with higher WMC are more likely to experience and express more negative emotion in response to negative emotional stimuli (Schmeichel et al., 2008).

Mindfulness training has demonstrated improvements in WMC (e.g., van Vugt \& Jha, 2011) and emotion regulation (e.g., Goldin \& Gross, 2010) as well as improvements in psychological well-being (e.g., Farb et al., 2010; Shapiro, Brown, \& Biegel, 2007). Jha et al. 
(2010) suggested that the improved emotional experience in a military population during their pre-deployment interval following mindfulness training was mediated by WMC. All participants completed the OSPAN and the Positive and Negative Affect Scale (PANAS; Watson, Clark, \& Tellegen, 1988) before and after one of the groups received eight weeks of mindfulness training. While they found that mindfulness training increased positive affect, mediational analysis indicated that the mindfulness training practice-related reductions in negative affect were indirect and mediated by WMC.

Previous studies of emotion regulation and WMC have reported that WMC corresponds to the ability to effectively regulate emotion, as opposed to changing emotions (i.e., positive emotions) for which people are not actively seeking change (Schmeichel et al., 2008).

Therefore, the authors suggested that the greater availability of WMC due to mindfulness training benefitted only negative affect because its expression and experience would require regulation whereas positive affect would not. This suggests a direct connection between WMC and emotion regulation. This study did not give specific instructions in regard to how to regulate emotions, nor did they inquire about the specific emotion regulation strategy the participant employed, which may be a limitation. Still, Jha et al. (2010) suggests WMC mediated the effect of mindfulness training on reducing negative affect because emotion regulation was required, which supports the potential of utilizing performance on a working memory task as a measure of successful emotion regulation.

\section{Mindfulness, Emotion Regulation, and Physiological Reactivity}

The measure of physiological reactivity has been employed in many experimental studies as an indicator of emotional response to emotion induction stimuli (e.g., Erisman \& Roemer, 2010; Smith et al., 2011). For example, heart rate variability (HRV), the coupling of heart rate 
and respiration, provides a reliable measure of parasympathetic nervous system activation (Berntson et al., 1997), which has recently been shown to underlie crucial aspects of emotion regulation. Heart rate is largely under the inhibitory control of the parasympathetic system, and this inhibition varies across the respiratory cycle. As mechanical stretch receptors in the lungs respond to inhalation, inhibition of heart rate decreases resulting in increased heart rate. Heart rate then slows during exhalation as inhibition returns (Berntson et al., 1997). The pattern of variability in heart rate and changes in respiration is called respiratory sinus arrhythmia, and is measured as HRV. Greater changes in heart rate across the respiratory cycle (i.e., increases in HRV) indicate greater parasympathetic activation, which is evoked by efforts to regulate emotion and behavior (Smith et al., 2011).

Smith et al. (2011) employed heart rate variability (HRV) as an indicator of both emotion regulation capacity (resting levels of $\mathrm{HRV}$ ) and emotion regulation effort (transient increases in HRV). They found that higher resting levels of HRV were correlated with the report of greater marital quality, suggesting that a greater capacity for emotion regulation is associated with adaptive functioning necessary in close relationships. Furthermore, after a negative marital interaction task where participants were asked to use negative adjectives to describe their partner who would then respond to their spouse's comments, participants demonstrated increased HRV, indicating effortful emotion regulation. Smith and colleagues (2011) suggest that HRV is a reliable measure of emotion regulation capacity (i.e., resting HRV) and effort (i.e., HRV during or following emotional provocation).

Skin conductance level (SCL), a measure of the electrical conductance of the skin, has been examined extensively in response to emotional stimuli. Because sweat glands are controlled by the sympathetic nervous system, and because skin conductance levels increase as moisture on 
the skin increases, SCL is used as an indication of psychological and physiological arousal (Ortner, Kilner, \& Zelazo, 2007). For example, Moscovitch, Suvak, and Hofmann (2010) found that increases in SCL were significantly correlated with both increased negative affect and decreased positive affect. This suggests SCL is a reliable measure of emotional response. Several studies have used SCL to examine the effects of mindfulness training. For example, Ortner et al. (2007) found lower skin conductance levels (SCL) associated with less emotional interference in a cognitive task in participants who completed a 7-week mindfulness training as compared to participants who received only relaxation training. SCL appears to be a reliable measure of emotional responsivity.

Erisman and Roemer (2010) conducted an experimental study examining the relationship between a brief mindfulness intervention and emotion regulation utilizing self-report and physiological measures. Participants were a sample of 30 individuals who reported high levels of difficulties in emotion regulation in response to a questionnaire, who were then randomly assigned to either the mindfulness or control condition. Two physiological measures, heart rate and skin conductance, were recorded continuously during the emotion induction and recovery periods. Two self-report measures were completed immediately after each emotion induction: the Positive and Negative Affect Scale (PANAS; Watson, Clark, \& Tellegen, 1988) which measures current emotional state, and the State Difficulties in Emotion Regulation Scale (DERSS; McLaughlin, Mennin, \& Farach, 2007) that assesses current or state emotion dysregulation as opposed to dispositional emotion dysregulation. The Toronto Mindfulness Scale (TMS; Lau, Bishop, Segal, Buis, Anderson, Carlson et al., 2006), designed to capture the extent to which a participant experiences heightened awareness, was administered as a manipulation check immediately following the brief mindfulness training (Erisman \& Roemer, 2010). 
Erisman and Roemer (2010) measured participants' baseline heart rate (HR) and skin conductance levels (SCL) during a 5 minute rest period and then had them view three film clips: negative (8-min clip from Saving Private Ryan), positive (155-sec clip from When Harry Met Sally), and mixed (125-sec clip from Life is Beautiful). Participants completed self-report measures of affect and emotion regulation immediately following each clip. Next, those in the mindfulness condition listened to a recorded 10-minute mindfulness intervention while those in the control group listened to two educational excerpts from public radio and completed a word search puzzle, both then completing the TMS as a manipulation check. The procedure for the second series of film clips was similar to the first, but different excerpts were used from the negative and mixed film selections. Participants in the mindfulness condition heard an additional brief mindfulness prompt before each clip: "If you notice any emotions during the film, try to just acknowledge and accept them as they are, without trying to change your experience in any way" (Erisman \& Roemer, 2010, p. 76). At the completion of the study, participants completed a second TMS to assess state mindfulness.

Erisman and Roemer (2010) found that the participants in the mindfulness condition reported higher levels of positive affect post-intervention after viewing the positive film than those in the control condition, indicating that mindfulness may facilitate increased engagement with positive emotional experiences. However, in regard to emotional response to the negative film clip, there were no significant differences between conditions. It is possible that negative film clips simply did not induce sufficient negative affect to require regulation. The authors cite the possibility that the film clips were not sufficient for emotional induction as a limitation. This would be consistent with the assertion of Jha et al. (2010) that reductions in negative emotions associated with mindfulness training would require emotion regulation, whereas 
changes in positive emotions, which individuals do not seek to change or eliminate, do not actually require regulation. Future research with proven and standardized emotional induction stimuli would shed light of the lack of significant differences between conditions in response to the post-intervention negative film clip.

In regard to the affectively mixed clip, participants in the mindfulness condition reported less difficulty regulating their emotions (i.e., DERS-S scores) and significantly less negative affect immediately following the clip than those in the control group, suggesting that mindfulness might facilitate regulation by reducing initial reactivity to emotionally complex stimuli. In addition, the results from this study suggest that the brief mindfulness intervention successfully induced mindfulness in an experimental situation as indicated by participant report of higher levels of decentering on the state mindfulness measure than participants in the control condition. This study provides important preliminary support for the role of mindfulness in the facilitation of adaptive responses to emotional experiences (Erisman \& Roemer, 2010).

No significant differences emerged between conditions on the physiological measures of heart rate and skin conductance at any assessment point. Erisman and Roemer (2010) suggest that the film clips employed for emotional induction may not have been sufficiently emotionally evocative. This possibility could be addressed through the use of emotion induction stimuli that have been standardized on the basis of normative ratings with respect to valence and arousal. Second, they suggest that this may be evidence that mindfulness differentially impacts an individual's subjective experience of emotion (i.e., report of less negative affect after the mixed clip) and their physiological arousal. Erisman and Roemer (2010) recommend that this be explored further in future research. 
Erisman and Roemer (2010) reported several additional limitations to their study that could be addressed in future research. First, the difference in state emotion dysregulation between the two conditions after viewing the mixed clip approached significance with a medium to large effect; thus, the authors suggest there may have been an issue with power. Second, participants in the mindfulness condition showed a significant increase in their level of mindfulness according to scores on the TMS pre- and post-intervention. However, the level of mindfulness achieved in this study is lower than TMS scores of clients receiving longer mindfulness interventions in clinical settings (Baer et al., 2004). In order to increase the external validity of the findings, the authors recommend increasing the mindfulness intervention to 15 minutes (instead of 10), which has been shown to significantly increase the level of state mindfulness in participants (Baer et al., 2004), and would make results more generalizable to clinical populations in which mindfulness is employed. The authors suggest, thirdly, that future studies should include a range of stimuli (e.g., pictures, individualized vignettes) to provide ecologically valid emotional experience which might elicit stronger emotions, and thereby greater efforts to regulate those emotions. Finally, the authors suggested that non-self-report indicators of emotional responding, such as performance on cognitive tasks, would also further our understanding (Erisman \& Roemer, 2010).

\section{Present Study}

Emotion regulation has been shown to be an essential component of psychological health (Gross, 1998), and emotion dysregulation has been associated with various forms of psychological distress, imbalance, and inflexibility (Gross, 2002) which may result in anxiety (Olatunji et al., 2007), depression (Ehring et al., 2010; Teasedale et al., 2000), maladaptive functioning in close relationships (Smith et al., 2011) and negative automatic thoughts (Frewen 
et al., 2007). Several lines of research have suggested that mindfulness interventions, which have been shown to improve psychological flexibility and functioning (e.g., Shapiro et al., 2007; Perlman et al., 2010), do so by providing a way to cultivate effective emotion regulation. Previous research supports the notion that emotion regulation mediates the relationship between mindfulness and its positive effects (e.g., decreased negative affect), however there is still much to be learned.

To further examine the supposition that emotion regulation mediates the relationship between mindfulness and psychological well-being (i.e., increased positive and decreased negative affect), the present study will take several steps. First, the study of mindfulness and emotion regulation has often relied solely on self-report measures. The use of physiological measures, like electroencephalogram (EEG) to observe frontal brain asymmetry (e.g., Davidson et al., 1990; Tomarken et al., 1990), heart rate variability (Smith et al., 2011) and skin conductance (Ortner et al., 2007) would provide more objective measures of emotion regulation. Second, mindfulness interventions have been correlated with improvements in working memory capacity (WMC), and this improvement has been shown to result in reductions in negative affect (Jha et al., 2010). Furthermore, increased WMC has been shown to improve emotion regulation and, thus, may mediate the relationship between mindfulness and improved emotional responding. Therefore, performance on the AOSPAN (a working memory task) will also provide an objective representation of an individual's effort to regulate emotion. Third, Erisman and Roemer (2010) recommend that a longer mindfulness intervention than theirs be used to make results more generalizable to clinical settings, which the current study will institute. Finally, Erisman and Roemer (2010) suggested that their lack of significant physiological results may be due to their choice of emotion induction; the current study will employ a range of stimuli that 
have been standardized on the basis of normative ratings with respect to valence and arousal to ensure the elicitation of stronger emotions.

Despite preliminary support for psychological well-being due to mindfulness training being mediated by emotion regulation, to the awareness of this investigator, no study has investigated this question with the simultaneous use of physiological measures and a working memory task. Thus, the present study employed physiological measures and a working memory task in addition to self-report measures to seek a more accurate understanding of the relationship between brief mindfulness training and the experience and regulation of emotion.

\section{Hypotheses and Proposed Data Analyses}

Hypothesis 1: It was hypothesized that mindfulness training would increase the subjective, neurological, and physiological experience of mindfulness as compared to the Control group. Specifically, it was predicted that the Mindfulness group would demonstrate greater mindfulness post intervention, as measured by the Toronto Mindfulness Scale (TMS), as compared to the control group. To test this hypothesis, an independent sample t-test was conducted with group (i.e., mindfulness versus control) as the independent variable and the mean of TMS scores as the dependent variable. Likewise, it was hypothesized that the mindfulness induced by training would last throughout the experimental session. Specifically, the scores on the Toronto Mindfulness Scale of the Mindfulness group would not differ significantly between Time 1 (immediately after the intervention/control) and Time 2 (at the very end of the experimental session). To test this hypothesis, a repeated measures t-test was conducted with time as the independent variable and the scores on the TMS as the dependent variable.

Hypothesis 2: It was hypothesized that a relationship between mindfulness training and emotional well-being would be demonstrated. Specifically, after emotion induction (positive or 
negative), emotional well-being would be significantly better (i.e., lower negative and higher positive scores on the Positive and Negative Affect Schedule; Watson, Clark, \& Tellegen, 1988) in the Mindfulness group as compared to the Control group. Likewise, the Mindfulness group would demonstrate greater Left $>$ Right frontal brain activation than the Control group after the emotional stimuli presentation as determined by the EEG laterality coefficients. Furthermore, the Mindfulness group would demonstrate lower skin conductance levels (SCL) than the Control group after the emotional stimuli presentation. To test this hypothesis, three MANOVAs were conducted with Group (Mindfulness vs. Control) and Induction (Positive or Negative) as the independent variables and the mean outcome variable (PANAS positive and negative scores, EEG laterality coefficients, or SCL) as the dependent variable.

Hypothesis 3: It was hypothesized that a relationship between mindfulness training and emotion regulation would be demonstrated. Specifically, it was predicted that participation in mindfulness training will result in greater efforts to regulate emotion as measured by heart rate variability (i.e., greater heart rate variability equals greater emotion regulation) during and after the emotional stimuli presentation as compared to the Control group. Likewise, the Mindfulness group would demonstrate lower scores on the DERS-S (measure of state emotion dysregulation) than the Control group after the emotional stimuli presentation. Furthermore, it has been previously demonstrated that working memory capacity has a relationship with emotion regulation (Jha et al., 2010). Therefore, it was hypothesized that a relationship between mindfulness training and emotion regulation would also be demonstrated by performance on a working memory task. Specifically, it was predicted that participation in mindfulness training will result in higher scores on a working memory task (AOSPAN) after the emotional stimuli presentation as compared to the Control group. To test this hypothesis, three MANOVAs were 
conducted with Group (Mindfulness vs. Control) and Induction (Positive or Negative) as the independent variables and the mean outcome variable (heart rate variability, DERS-S scores, or AOSPAN scores) as the dependent variable.

Hypothesis 4: It was hypothesized that the relationship between mindfulness training and emotional well-being, as measured by frontal brain asymmetry, PANAS scores, and SCL, would be mediated by emotion regulation, as measured by heart rate variability and the DERS-S, and represented by working memory capacity (AOSPAN) scores. To test for mediation, an SPSS macro developed by Preacher and Hayes (2008) was employed, which tests for direct and indirect effects when multiple mediators are predicted to demonstrate an effect on the outcome variable or variables. The rationale for their method is that indirect effects can be present when total effects are absent; therefore, even if the independent variable fails to demonstrate change in the dependent variable, mediation is still possible (Hayes, 2009). The Preacher and Hayes method has advantages related to its use of bootstrapping, a nonparametric resampling procedure that does not require normality of the sampling distribution. Further advantages to the method include reduction in the likelihood of Type 1 error due to minimization of the number of inferential tests and multiple mediators can be tested simultaneously (Preacher \& Hayes, 2008). In addition to the bootstrapped confidence intervals provided by the Preacher and Hayes (2008) model, the SPSS macro described above also provides the path coefficients that would be examined as part of a causal model. Statistically significant path coefficients may indicate a significant relationship between two variables but are not proof of a causal connection. Also, the model summary presented for the path coefficients, which represents how well the independent variable and mediational variable together predict the dependent variable, can be statistically significant without indicating a mediational relationship based on the significance/non- 
significance of other path coefficients. Therefore, no hypotheses are made regarding these coefficients as the bootstrapping confidence intervals of the Preacher and Hayes model (2008) provide advantages over the causal model. However, where this additional data proved statistically significant, it was reported to bring greater understanding to the relationships between variables.

\section{Method}

\section{Inclusion and Exclusion Criteria}

Studies examining emotion regulation often disqualify participants reporting current use of psychotropic medication (e.g., Papousek et al., 2012; Goldin \& Gross, 2010). Participants were queried regarding the current use of psychotropic medication, and the data of 6 participants reporting current use (i.e., Focalin, Klonopin, Vyvanse, Zoloft, Celexa, and Ativan) was excluded. In addition, the data of participants demonstrating low effort was excluded. Low effort was defined as obvious inattention to items answered on self-report measures indicated by the selection of the same rating number throughout. Low effort may also be indicated by the direct report of participants following completion of the study (e.g., participant stated that they “didn't give it my best"). The data of two participants was excluded for low effort. Therefore, though a total of 78 participants completed the experimental process, only 70 participants' data were used for analyses.

\section{Participants}

Seventy undergraduate students (51 women and 19 men; $73 \%$ and $27 \%$, respectively) who had enrolled in Psychology 101 classes at the University of South Carolina Aiken participated in the study and received class credit for their participation. A description of the study stating that participants would observe emotionally provocative pictures and sounds and 
perform a working memory task was posted on the Psychological Experiment board where students signed-up for participation in the study. Of the 70 students, 46 were Caucasian (66\%), 20 African-American (29\%), 2 Asian (3\%), 1 Native American (1\%), and 1 African (1\%). The average age of the participants was $19.31(S D=2.33)$

\section{Stimuli}

Sounds from the international affective digitized sound system (IADS; Bradley \& Lang, 1999) and images from the international affective picture system (IAPS; Lang, Bradley, \& Cuthbert, 2008) were utilized to create the emotion induction stimuli. The international affective picture system (IAPS; Lang, Bradley, \& Cuthbert, 2008) is a well-established source of visual images for eliciting affective responses in psychological research. The IAPS stimuli are standardized on the basis of normative ratings with respect to valence, arousal, and dominance and have been corroborated by several lines of research (e.g., Colden, Bruder, \& Manstead, 2008). The international affective digitized sound system (IADS; Bradley \& Lang, 1999) provides a set of acoustic emotional stimuli for use in psychological research which has also been standardized with respect to valence, arousal and dominance. Both the IADS and the IAPS are distributed by the Center for Emotion and Attention (CSEA) at the University of Florida.

The present study presented individuals with either a positive or negative series of emotional stimuli including both pictures and sounds. For each series, 75 images (see Appendix A) and 25 sound clips (see Appendix B), matched on valence (positive range $=6.05-8.05$; negative range $=1.95-3.95)$ and arousal (range for pictures $=4.95-5.95$; range for sounds $=$ 4.95 - 6.95) were selected. Pictures ( 2 second presentation each) and sounds (6 second presentation each) were presented in a randomized order. The images and sound clips were 
presented with a .5 second inter-stimulus interval, consisting of the presentation of a fixation cross, making each series approximately 6 minutes long.

\section{Measures}

Demographic questionnaire. Demographic information (e.g., gender, race, ethnicity, exclusion criteria) was gathered utilizing a questionnaire developed by the author (see Appendix C for this measure).

Difficulties in Emotion Regulation Scale (DERS; Gratz \& Roemer, 2004). The DERS is a 36-item, self-report measure developed to assess clinically relevant difficulties in emotion regulation. Participants were asked to indicate how often the items applied to themselves, with responses ranging from 1 to 5 , where 1 is almost never (0-10\%), 2 is sometimes (11- 35\%), 3 is about half the time (36-65\%), 4 is most of the time (66-90\%), and 5 is almost always (91100\%). Higher scores indicated greater difficulties in emotion regulation (i.e., greater emotion dysregulation). The DERS items reflect difficulties within the following dimensions of emotion regulation: (a) awareness (Lack of Emotional Awareness) and understanding of emotions (Lack of Emotional Clarity); (b) acceptance of emotions (Nonacceptance of Emotional Responses); (c) the ability to engage in goal-directed behavior (Difficulties Engaging in Goal Directed Behavior), and refraining from impulsive behavior (Impulse Control Difficulties), when experiencing negative emotions; and (d) access to emotion regulation strategies perceived as effective (Limited Access to Emotion Regulation Strategies). The final dimension reflects an attempt to measure the flexible use of appropriate strategies to modulate emotional responses. Gratz and Roemer (2004) indicate that the DERS has high internal consistency $(\alpha=.93)$ and good test-retest reliability (.88) and construct validity (.69). This measure was used in the present 
study to assess participants' dispositional difficulties in emotion regulation (see Appendix D for this measure).

Mindfulness Attention Awareness Scale (MAAS; Erisman \& Roemer, 2010). The MAAS is a 15-item self-report measure of trait characteristics of mindfulness. Specifically, the MAAS considers an individual's inherent ability to pay attention to the present moment and to maintain nonjudgmental awareness of whatever is experienced in the present moment. Research has confirmed that the MAAS is a single factor scale structure (Brown \& Ryan, 2003; Carlson \& Brown, 2005). Internal consistency has been found to range from .80 to .90 (Erisman \& Roemer, 2010). The MAAS has demonstrated high test-retest reliability (.81), and discriminant and convergent validity (.70; Brown \& Ryan, 2003). The trait MAAS was used in the present study to assess the participants' dispositional mindfulness (see Appendix E for this measure).

Positive and Negative Affect Schedule (PANAS; Watson et al., 1988). The PANAS is a 20-item self-report measure that assesses an individual's positive and negative affect at a given point in time. Twenty different feelings and emotions are listed (10 positive and 10 negative) and individuals rate items on a 5-point Likert scale from 1 (very slightly or not at all) to 5 (extremely). The PANAS has demonstrated good internal consistency (.86 - .90 for Positive affect and $.84-.87$ for Negative affect) and moderate concurrent validity (.51 - .74; Watson et al., 1988). This measure was used in the present study to assess the participants' positive and negative affect after the emotion induction (see Appendix F for this measure).

State Difficulties in Emotion Regulation Scale (DERS-S; McLaughlin et al., 2007). The intention was to use this state measure of the DERS (DERS-S), a self-report measure, to assess current emotion dysregulation as opposed to dispositional emotion regulation. However, there were 10 items out of 36 in the measure used for the current study that had slight differences 
in wording. These differences were related to changes adapting some questions to more greatly reflect the present moment [e.g., I am embarrassed for feeling this way (McLaughlin et al., 2007) vs. I become embarrassed for feeling this way (current study)]. Participants indicated how they felt "in the present moment" on a 5-point Likert scale ranging from 1 (not at all) to 5 (completely). The measure consisted of the same 6 subscales as the DERS: awareness, clarity, acceptance, goals, impulse and limited access to strategies. The state version of the DERS (DERS-S) demonstrated good internal consistency (.81; McLaughlin et al., 2007). In the current study, the measure utilized also demonstrated good internal consistency (awareness .87, clarity .80 , acceptance .90 , goals .83, impulse .76, and limited access to strategies .79). The DERS-S was used in the current study to determine the individual's state difficulties in emotion regulation after the presentation of emotional stimuli (see Appendix $G$ for this measure).

Toronto Mindfulness Scale (TMS: Lau et al., 2006). The TMS is a state measure of mindfulness consisting of 13 items assessing two factors (Curiosity and Decentering) with the intent of capturing the extent to which participants experience feelings of increased awareness. The Curiosity factor indicates a quality of awareness that consist of openness and curiosity; the Decentering factor indicates the ability to be aware of one's thoughts and feelings without becoming entangled in them. Internal consistency is good for the Curiosity scale (.90) and adequate for the Decentering scale (.69; Erisman \& Roemer, 2010). This measure was used in the present study to determine the participants' state mindfulness after the mindfulness intervention/control period and again at the end of the experimental session (see Appendix $\mathrm{H}$ for this measure).

Physiological reactivity. Heart rate variability (HRV) was quantified from a continuous recording of the electrocardiogram signal (ECG), which also provides heart rate. Heart rate 
variability refers to the coupling of heart rate and respiration, in that the heart rate varies, repeatedly rising and falling as a person breathes in and out, and provides a well-validated measure of parasympathetic nervous system activation (Smith et al., 2011). In other words, the greater the changes in heart rate during a respiratory cycle, the greater the parasympathetic activation, which has been shown to underlie key aspects of emotion regulation and expression (Smith et al., 2011). The experimenter placed electrodes on the participants' right and left inner ankles and on their right inner forearm to record ECG continuously throughout the experimental session using the Biopac encoder unit and AcqKnowledge 3.9 software (Biopac Systems, Goleta, CA) with a sampling rate of 1,000 samples per second. The ECG data was checked and edited for artifacts, and the HRV Vagal ratio was then calculated from ECG on the basis of interbeat intervals using the Biopac HRV analysis.

Skin conductance levels (SCLs) were continuously assessed throughout the experimental session using the Biopac encoder unit and AcqKnowledge 3.9 software (Biopac Systems, Goleta, CA) with a sampling rate of 1,000 samples per second. The experimenter placed electrodes on the right and left sides of the palm of participants' right hand to record SCLs. Mean levels of skin conductance were analyzed with AcqKnowledge 3.9 software.

Frontal brain asymmetry. EEG was continuously assessed throughout the experimental session using the Biopac encoder unit and AcqKnowledge 3.9 software (Biopac Systems, Goleta, CA). Electrodes were attached at the ventrolateral positions F7 and F8 (Stern, Ray, \& Quigley, 2001). All EEG data was inspected visually and muscle artifacts were eliminated using the Biopac Connect Endpoints mathematical function. Utilizing the common approach in the field (Papousek et al., 2012), the alpha frequency band $(8-13 \mathrm{~Hz})$ was averaged across artifact free intervals. To determine frontal brain asymmetry, the mean alpha activation for each hemisphere 
was inputted into the calculation for a laterality coefficient (LC), to relate the left and right hemisphere data $(\mathrm{LC}=\mathrm{R} / \mathrm{L})$. Values greater than 1 indicate higher activity in the left than in the right hemisphere (Moyer et al., 2011). EEG was measured throughout the experiment to determine if frontal brain asymmetry corresponds with the results of other measures.

Working memory task. The automated version of the operation span task (AOSPAN; Unsworth, Heitz, Schrock, \& Engle, 2005), which takes approximately 20 minutes to complete and requires the participant to solve mathematical problems while performing a short-term memory test, was devised to measure individual differences in working memory capacity (WMC). It is shown that this version of operation span task (OSPAN) correlates well with other measures of WMC and has both good internal consistency (.78) and test-retest reliability (.83; Unsworth et al., 2005). In addition, the AOSPAN was shown to load on the same factor as two other WM measures. Previous research has indicated that higher scores on a WMC task are correlated with lower emotion dysregulation (i.e., Jha et al., 2010). Therefore, the proposed study utilized performance on the AOSPAN task as an indicator of the capacity for emotion regulation. The AOSPAN task was completed by the individual following the presentation of the emotional stimuli.

\section{Experimental Manipulation}

The 15-minute mindfulness intervention was adapted from Erisman and Roemer's (2010) 10-minute intervention with extensions of the two mindfulness exercises. Erisman and Roemer took their mindfulness intervention from exercises often used in clinical application (e.g., Acceptance and Commitment Therapy: Hayes et al., 1999; MBSR: Kabat-Zinn, 1990). The mindfulness intervention was recorded and presented in E-Prime, and described the concept of mindfulness followed by an experiential exercise in mindfulness. The recording then described 
the way in which mindfulness principles apply to emotional experiences followed by another experiential exercise in which participants are mindful of their emotions (see Appendix $G$ for the details of the intervention).

Participants in the control condition listened to a recording of a 15 minutes excerpt from a gardening program with neutral content. This is similar to the neutral material that Erisman and Roemer (2010) employed for their control condition.

\section{Procedure}

After the participant read and signed a written informed consent (see Appendix $\mathbf{J}$ for the details), he or she was seated alone in an examination room in a comfortable chair in front of a table that has a 19" computer monitor on it, a standard keyboard, and a mouse. The participant then completed trait measures of mindfulness and emotion dysregulation and a demographic questionnaire. Electrodes for the physiological measures were then attached, and the remainder of the study was completed on the computer. Participants were randomly assigned to either the mindfulness training group or the control group as well as randomly assigned to receive either a positive or negative emotion induction. Participants were instructed to be as still as possible while EEG, HRV and SCL were recorded in a 2-minute rest period. Then participants completed practice for the working memory task to be performed later before they were lead through either a 15-minute mindfulness intervention or listened to 15 minutes of a neutral program. Participants then completed the TMS presented on the computer using E-Prime software.

Depending on their randomly assigned group, each participant was then presented with a positive or negative series of emotional stimuli with E-Prime software. Participants in the mindfulness condition read an additional brief mindfulness prompt preceding the clip: "If you notice any emotions during the presentation of pictures and sounds, try to just acknowledge and 
accept them as they are, without trying to change your experience in any way." Upon completion of the presentation of emotional stimuli, each participant completed the PANAS and the DERS-S in E-Prime and then the AOSPAN task. Physiological measures were recorded throughout the experiment. Once the AOSPAN task was completed, participants completed the TMS again, also presented with E-Prime. Once the experimental session was complete, participants were debriefed about the study and offered contact information for the counseling center on the University's campus in the event that the experiment had been distressing.

\section{Results}

\section{Preliminary Analyses}

While 70 participants completed self- report measures, 14 participants' physiological data was eliminated due to physiological recording equipment error. In order to determine whether there were any differences between the Mindfulness and Control groups on trait mindfulness or trait emotion regulation prior to the intervention, independent samples t-tests were performed with Group as the IV and either MAAS scores (trait mindfulness) or DERS scores (trait emotion dysregulation) as the DV. No significant differences were discovered between groups for the MAAS, $t(68)=-.57, p=.33$, with the mindfulness group $(M=3.88, S D=.90)$ reporting similar trait mindfulness as the control group $(M=4.00, S D=.74)$. Also, no significant differences were revealed between groups on the DERS, $t(68)=-.84, p=.72)$, with the mindfulness group $(M=$ 72.34, $S D=19.00)$ reporting similar trait emotion dysregulation as the control group $(M=76.13$, $S D=18.68)$. In addition, independent samples t-tests were performed to determine if any differences existed between groups on baseline measures of skin conductance levels (SCL), heart rate variability (HRV), and frontal brain asymmetry (EEG). No significant differences appeared 
between groups on these variables: SCL, $t(55)=1.27, p=.21, \mathrm{HRV}, t(55)=-.14, p=.89$, and frontal brain asymmetry, $t(55)=.30, p=.77$.

\section{Mindfulness}

Toronto Mindfulness Scale (TMS). Hypothesis One predicted that participants in the Mindfulness condition would report greater mindfulness than those in the Control condition. A one-way ANOVA was conducted with Group (Mindfulness or Control) as the IV and state mindfulness scores (TMS total) as the DV. Results indicated a significant difference between groups, $F(1,68)=4.527, p=.037$, with the Mindfulness group demonstrating higher TMS scores $(M=43.34, S D=8.77)$ than the Control group $(M=38.78, S D=9.12)$ post-intervention. The TMS also contains two subscales, Curiosity and Decentering. To further explore the differences between groups, one-way ANOVAs were conducted for Decentering and Curiosity. Results indicated a significant difference between groups for Decentering, $F(1,68)=5.38, p=.02$, with the Mindfulness group demonstrating significantly greater Decentering $(M=23.21, S D=4.77)$ than the Control group $(M=20.63, S D=4.50)$ but not Curiosity, $F(1,68)=1.96, p=.17$. However, the Mindfulness group did tend to have higher Curiosity scores $(M=20.13, S D=$ 5.72) than the Control group $(M=18.16, S D=6.07)$. Thus, the first part of Hypothesis One was largely supported.

Hypothesis One also predicted that mindfulness training would increase mindfulness sufficiently to maintain its effects through the end of the experimental period. Specifically, no significant change would be demonstrated by the Mindfulness group in TMS scores between post-intervention (Time 1) and the completion of the experimental period (Time 2). A paired samples $t$-test was conducted with Mindfulness group TMS total scores at Time 1 and Time 2 . Results indicated a significant difference between Time 1 and Time $2, t(37)=2.053, p=.05$, 
with TMS total scores at Time $2(M=40.37, S D=9.92)$ being significantly lower than Time 1 $(M=43.34, S D=8.77)$. A paired-samples t-test comparing the TMS subscale Decentering at Time 1 and Time $2, t(37)=2.06, p=.05$, indicated a significant difference with Time $2(M=$ 21.61, $S D=5.12)$ being lower than Time $1(M=23.21, S D=4.77)$. However, a paired- samples t-test comparing the TMS Curiosity subscale at Time 1 and Time 2 indicated no significant difference, $t(37)=1.53, p=.14$. Therefore, the second part of Hypothesis One was partially supported (see Table 1 for all TMS means).

Though no predictions were made regarding differences of TMS scores at Time 2 between conditions, additional analyses were performed to explore these possibilities. A MANOVA was conducted with Group and Induction as the IVs and TMS total scores and Decentering and Curiosity subscale scores as the DVs. No significant main effects were revealed for Group, $F(1,66)=.09, p=.76$, or Induction, $F(1,66)=.27, p=.61$, or any significant interactions, $F(1,66)=.01, p=.94$.

Exploratory analyses related to hypothesis one. In the interest of providing further support for the assertion that the mindfulness intervention successfully increased mindfulness, HRV during the completion of the 15-minute recording (Time 2; see Figure 1) was analyzed. Previous research (e.g., Tang et al., 2009) suggests that HRV increases during mindfulness training, especially in novices, therefore a one-way ANOVA was conducted with Group as the IV and HRV during the completion of the intervention as the DV. Results indicated a significant difference between groups, $F(1,55)=3.90, p=.05$, with the Mindfulness group $(M=.43, S D=$ .19) demonstrating greater HRV than the Control group $(M=.33, S D=.18)$. This is consistent with the state mindfulness scores, and these results suggest that the brief mindfulness intervention successfully increased mindfulness. 
To further support the prediction that the mindfulness intervention successfully increased mindfulness, frontal brain asymmetry at the completion of the 15 minute recording (Time 2) was analyzed. Previous research (Keune, Bostanov, Hautzinger, \& Kotchoubey, 2013; Moyer et al., 2011) suggests that $L>R$ frontal brain activation is a pattern indicative of approach-motivation and the capacity for emotion regulation characteristic of mindfulness. To determine frontal brain asymmetry, the mean alpha activation for each hemisphere was inputted into the calculation for a laterality coefficient $(\mathrm{LC})$, to relate the left and right hemisphere data $(\mathrm{LC}=\mathrm{R} / \mathrm{L})$. Values greater than 1 indicate higher activity in the left than in the right hemisphere (Moyer et al., 2011). Next, a one-way ANOVA was conducted with Group as the IV and the EEG laterality coefficient as the DV. Results indicated a significant difference between groups $F(1,55)=6.14, p=.02$, with the Mindfulness group $(M=1.26, S D=.69)$ demonstrating greater $\mathrm{L}>\mathrm{R}$ frontal brain activation than the Control group $(M=.86, S D=.47)$. This is consistent with the self-reported state mindfulness as well as the HRV results, further suggesting that the brief mindfulness training successfully increased mindfulness.

\section{Emotional Well-being}

Positive affect (PANAS positive scores). Hypothesis Two predicted that, after emotion induction (i.e., positive or negative), emotional well-being would be significantly better (i.e., higher positive affect) in the Mindfulness group as compared to the Control group. A two-way ANOVA was conducted with Group and Induction as the IVs and positive affect (PANAS positive scores) as the DV. Results indicated a main effect of Group on positive affect, $F(1,66)=$ $4.78, p=.03$, with the Mindfulness group $(M=30.11, S D=8.35)$ reporting significantly greater positive affect than the Control group $(M=25.34, S D=8.430)$ regardless which induction they received. Results indicated a main effect for Induction for positive affect, $\mathrm{F}(1,66)=5.14, p=.03$, 
with the positive induction $(M=29.83, S D=8.83)$ resulting in greater positive affect than the negative induction $(M=25.40, S D=7.80)$. No significant interaction between Group and Induction was indicated. These results support the prediction that mindfulness training would result in greater positive affect as compared to the Control group.

Negative affect (PANAS negative scores). Hypothesis Two also predicted that, after emotion induction (i.e., positive or negative), negative affect would be significantly lower in the mindfulness group as compared to the control group. A two-way ANOVA was conducted with Group and Induction as the IVs and negative affect (PANAS negative scores) as the DV which indicated no significant main effect for Group, $F(1,66)=.04, p=.84$ and a main effect for Induction, $F(1,66)=7.87, p=.01$. However, a significant interaction of Group and Induction was found, $F(1,66)=4.93, p=.03$. Specifically, the Control group experiencing the Positive Induction $(M=15.71, S D=2.56)$ or the Negative Induction $(M=16.44, S D=5.78)$, as well as the Mindfulness group after the Positive Induction $(M=13.19, S D=3.86)$ reported similar negative affect; however, the Mindfulness participants receiving the Negative Induction $(M=$ $19.47, S D=7.14$ ) reported significantly higher negative affect. These results are the opposite of what was predicted by Hypothesis Two.

Frontal brain asymmetry (EEG). Hypothesis Two also stated that the Mindfulness group would demonstrate greater L > R frontal brain activation than the Control group during emotion induction, but this prediction was not supported. A MANOVA was conducted with Group and Induction as the IVs and EEG laterality coefficients as the DV, which resulted in no main effects for Group, $F(1,55)=1.19, p=.28$, or Induction, $F(1,55)=.30, p=.59$, and no significant interaction, $F(1,55)=.001, p=.98$. However, the laterality coefficients moved in the 
predicted direction, with the Mindfulness group $(M=1.02, S D=.68)$ demonstrating $\mathrm{L}>\mathrm{R}$ frontal brain asymmetry and the Control group $(M=.84, S D=.43)$ demonstrating $\mathrm{R}>\mathrm{L}$.

Skin conductance (SCL). Hypothesis Two further stated that the Mindfulness group would demonstrate lower skin conductance levels (SCLs) during the emotion induction than the Control group regardless of the type of emotion induction, but this prediction was not supported. A two-way ANOVA with Group and Induction as the IVs and SCL during the induction as the DV indicated no significant differences or interaction effects $[F(1,53)=2.04, p=.16 ; F(1,53)=$ $.39, p=.54 ; F(1,53)=.142, p=.71$; for Group, Induction and interaction, respectively]. These results did not support the hypothesis that SCLs would be significantly lower for the Mindfulness group as compared to the Control group during the emotion induction.

\section{Emotion Regulation}

State Difficulties in Emotion Regulation Scale (DERS-S). Hypothesis Three stated that the Mindfulness group would demonstrate lower scores on the DERS-S (measure of state emotion dysregulation) than the Control group after the emotion induction. A MANOVA was conducted with Group and Induction as the IVs and emotion dysregulation subtest scores (DERS-S) as the DVs. Results indicated no significant differences for Group, and a main effect for Induction on Nonacceptance of Emotional Responses (NONACCEPTANCE), $F(1,66)=$ $4.97, p=.03$, with the Negative Induction group $(M=10.16, S D=5.05)$ reporting greater NONACCEPTANCE scores than the positive Induction group $(M=7.71, S D=3.66)$. There were no other main effects. A significant interaction emerged for Group and Induction regarding Difficulties Engaging in Goal-Directed Behavior (GOALS), $F(1,66)=4.94, p=.03$, Impulse Control Difficulties (IMPULSE), $F(1,66)=3.81, p=.05$, and Limited Access to Emotion Regulation Strategies (STRATEGIES), $F(1,66)=3.86, p=.05$. Participants in the Mindfulness 
group that experienced the negative emotion induction reported significantly greater emotion dysregulation on the three subscales GOALS, IMPULSE, and STRATEGIES $(M=11.65, S D=$ 3.72; $M=9.88, S D=4.06 ; M=14.53, S D=5.89$; respectively) than the other three conditions (Mindfulness positive, Control positive, and Control negative; see Table 2 for means). These results are the opposite of what was predicted in Hypothesis Three.

Further, a two-way ANOVA was conducted with Group and Induction as the IVs and the Total Emotion Dysregulation score as the DV. No significant differences were revealed for Group, $F(1,66)=.30, p=.58$, or Induction, $F(1,66)=2.01, p=.16$. In addition, while no significant interaction was revealed, $F(1,66)=3.08, p=.08$, the means were in the direction consistent with the interaction described regarding the three above subscales; such that participants in the Mindfulness group that experienced the negative emotion induction reported greater Total Emotion Dysregulation scores $(M=70.41, S D=21.03)$ than the other three groups (Mindfulness Positive: $M=56.86, S D=17.61$; Control Positive: $M=62.00, S D=12.97$; Control Negative: $M=60.56, S D=17.48)$. These results did not support the hypothesized prediction that the Mindfulness group would report significantly lower emotion dysregulation than the Control group after the emotion induction.

Heart rate variability (HRV). Hypothesis Three also stated that mindfulness training would result in greater efforts to regulate emotion as indicated by greater heart rate variability (HRV) during the emotion induction in the Mindfulness group compared to the Control group. A two-way ANOVA was conducted with Group and Induction as the IVs and HRV during the induction as the DV. The results indicated no significant differences for Group, $F(1,55)=.32, p$ $=.58$, or Induction, $\mathrm{F}(1,55)=.16, p=.70$, and no significant interaction, $F(1,55)=1.27, p=.27$. 
These results do not support the prediction that mindfulness training would result in greater HRV as compared to the Control group during the emotion induction.

Automated version of the operation span task (AOSPAN). Hypothesis Three stated that participants in the Mindfulness group would demonstrate better performance on a working memory task than those in the Control group. A two-way ANOVA was conducted with Group and Induction as the IVs and absolute AOSPAN scores as the DV. Results indicated that there were no significant differences for Group, $F(1,66)=.41, p=.53$, or Induction, $F(1,66)=.20, p=$ .66 , and no significant interaction, $F(1,66)=.01, p=.93$. Therefore, this prediction of Hypothesis Three was not supported.

\section{Mediation of Mindfulness Training Effects and Psychological Well-being by Emotion Regulation}

Hypothesis Four stated the relationship between Condition ( $\mathrm{X}_{1}$; Mindfulness or Control) and emotional well-being (Y; L > R frontal brain asymmetry, PANAS positive and negative scores, and SCLs) would be mediated by emotion regulation $\left(\mathrm{X}_{2}\right.$; heart rate variability, DERS-S scores, and AOSPAN scores). Several mediation analyses were performed utilizing the SPSS macro provided by Preacher and Hayes (2008) to conduct the mediational analysis.

DERS-S as a mediator. Completion of the DERS-S results in a total emotion dysregulation score as well as six subscale scores (NONACCEPTANCE, GOALS, IMPULSE, AWARENESS, STRATEGIES, and CLARITY). With seven mediators (the total plus the six subscales), three subscales were tested in one analysis, then three more and then the total emotion dysregulation score tested in another. These predicted mediators were investigated to judge their effect on four well-being variables: positive affect (PANAS positive scores), negative affect (PANAS negative scores), L > R frontal brain asymmetry (EEG), and SCLs. Therefore, 
three analyses were conducted for each well-being variable with condition (Mindfulness or Control) as the independent variable resulting in a total of 12 mediational models executed.

To assess the potential indirect effects of mindfulness training on psychological wellbeing through emotion regulation, three mediators, the DERS-S subscales NONACCEPTANCE, GOALS, and IMPULSE were entered into the first four models for the outcome variables listed above with Condition (Mindfulness or Control) as the IV. All potential mediators failed to demonstrate any significant total or specific indirect effects on $\mathrm{L}>\mathrm{R}$ frontal brain activation, SCLs, or positive and negative affect as all confidence intervals contained zeroes. Therefore, these results do not support the prediction of Hypothesis Four.

Despite finding no significant mediational effects, several significant path coefficients were found, which, though not sufficient to prove a causal connection, indicate significant relationships between two variables. A significant direct effect ( $b$ path) was indicated for the DERS-S subscale GOALS (mediator) on $\mathrm{L}>\mathrm{R}$ frontal brain activation, $b=-.12, p=.05$, on positive affect, $b=-.58, p=.04$, and on negative affect, $b=.39, p=.02$. This suggests that difficulty engaging in goal directed behavior has a significant negative correlation with positive affect, less $\mathrm{L}>\mathrm{R}$ frontal brain activation, and a significant positive correlation with negative affect.

A significant total effect of Condition ( $c$ path) was found, $c=-4.76, p=.02$, as well as a significant direct effect of Condition ( $c^{\prime}$ path), $c^{\prime}=-5.45, p=.01$ on positive affect. This suggests that the lack of mindfulness training (Control condition) including the effect of the mediators ( $c$ path) and without the effect of the mediators ( $c$ ' path) demonstrated a significant negative correlation with positive affect. In addition, for this model the ability of Condition and these three mediators to predict positive affect was significant, $F(4,65)=5.01, p=.001$. Further, 
for this model the ability of Condition and these three mediators to predict negative affect was significant, $F(4,65)=8.02, p=.0001$. While this indicates that together the effects of mindfulness training and self-reported difficulties with NONACCEPTANCE, GOALS, and IMPULSE may successfully predicted positive and negative affect, it does not suggest an order to these effects and therefore does not indicate a mediational relationship.

To continue to assess the potential indirect effects of mindfulness training on psychological well-being through emotion regulation, the next three mediators of the DERS-S (AWARENESS, STRATEGIES, and CLARITY) were entered into the next four models for the outcome variables ( $\mathrm{L}>\mathrm{R}$ frontal brain activation, SCLs, positive affect, negative affect) with Condition (Mindfulness or Control) as the IV. All potential mediators failed to demonstrate any significant total or specific indirect effects on any of the four outcome variables, as all confidence intervals contained zeroes. Therefore, these results do not support the prediction of Hypothesis Four.

Despite finding no significant mediational effects, again several significant path coefficients emerged. A significant direct effect ( $b$ path) was indicated for the DERS-S subscale STRATEGIES (mediator) on negative affect, $b=.54, p=.0002$. This suggests that limited access to emotion regulation strategies demonstrated a significant positive correlation with negative affect. For this model, the ability of mindfulness training and these three mediators to predict negative affect was significant, $F(4,65)=3.93, p=.006$. While this indicates that together the effects of mindfulness training and self-reported difficulties with AWARENESS, STRATEGIES, and CLARITY may successfully predict negative affect, it does not suggest an order to these effects and therefore does not indicate a mediational relationship. 
For this model, Condition demonstrated a significant total effect $(c=-4.76, p=.02)$, as well as a significant direct effect $\left(c^{\prime}=-4.96, p=.02\right)$ on positive affect. This suggests that a lack of mindfulness training including the effect of the mediators ( $c$ path) and without the effect of the mediators ( $c$ ' path) demonstrated a significant negative correlation with positive affect. In addition, for this model the ability of group and these three mediators to predict positive affect was significant, $F(4,65)=3.12, p=.02$. While this indicates that together the effects of mindfulness training and self-reported difficulties with AWARENESS, STRATEGIES, and CLARITY may successfully predict negative affect, it does not suggest an order to these effects and therefore does not indicate a mediational relationship.

Finally, to assess the potential indirect effects of mindfulness training on psychological well-being through emotion regulation, the last mediator of the DERS-S (Total score) was entered into the last four models for the outcome variables ( $\mathrm{L}>\mathrm{R}$ frontal brain activation, SCLs, positive affect, negative affect) with Condition as the IV. The potential mediator failed to demonstrate any significant total or specific indirect effects on any of the four outcome variables, as all confidence intervals contained zeroes.

Despite finding no significant mediational effects, again several significant path coefficients emerged. Regarding the effect of this mediator on negative affect, a significant direct effect ( $b$ path) was indicated, $b=.13, p=.0002$. This suggests that emotion dysregulation has a significant positive correlation with negative affect. For this model, the ability of group and this mediator to predict negative affect was significant, $F(4,65)=7.67, p=.001$. While this indicates that together the effects of mindfulness training and self-reported emotion dysregulation may successfully predict negative affect, it does not suggest an order to these effects and therefore does not indicate a mediational relationship. 
Regarding the effect of this mediator on positive affect, a significant direct effect ( $b$ path) was demonstrated, $b=-.17, p=.002$, indicating a significant negative correlation between emotion dysregulation and positive affect. For this model, Condition demonstrated a significant total effect $(c=-4.76, p=.02)$ as well as a significant direct effect $\left(c^{\prime}=-5.05, p=.01\right)$ on positive affect. This suggests that being in the Control condition, both including the effect of the mediators ( $c$ path) and without the effect of the mediators ( $c^{\prime}$ path), was associated with less positive affect. In addition, for this model the ability of Condition and this mediator to predict positive affect was significant, $F(4,65)=8.32, p=.0006$. While this indicates that together the effects of mindfulness training and self-reported emotion dysregulation may successfully predict positive affect, it does not suggest an order to these effects and therefore does not indicate a mediational relationship.

HRV as a mediator. To investigate the potential indirect effects of mindfulness training on psychological well-being through emotion regulation, HRV was measured during the emotion induction. It was entered into the model as a mediator to assess its effect on four outcomes (positive and negative affect, L > R frontal brain activation, and SCLs) with condition as the IV, for a total of four models tested. HRV as a mediator failed to produce any total or specific indirect effect on the four outcome variables as all confidence intervals contained zeroes.

Working Memory Capacity as a mediator. To investigate the potential indirect effects of mindfulness training on psychological well-being through emotion regulation, participants completed the AOSPAN, a working memory task. AOSPAN absolute scores were entered into the model as the mediator to examine their effects on four outcomes (positive and negative affect, L > R frontal brain activation, and SCLs) with Condition as the IV, for a total of four 
models tested. Working memory capacity as a mediator failed to produce any total or specific indirect effect on the four outcome variables as all confidence intervals contained zeroes.

\section{Discussion}

The past study of mindfulness, while demonstrating positive effects of mindfulness on psychological well-being (e.g., Goldin \& Gross, 2010), has been limited in its examination of the mechanisms of mindfulness (Davidson, 2010). Furthermore, research examining mindfulness has often relied solely on self-report measures which are cited as limitations in the literature (e.g., Sears \& Kraus, 2009). Therefore, the current study employed physiological measurement and a working memory task in addition to self-report to seek a greater understanding of the relationship between a brief mindfulness training and well-being through the exploration of emotion regulation as a mediator of this relationship.

The present study attempted to examine the ability of mindfulness to improve emotion regulation during an emotion induction and increase psychological well-being post-induction. Specifically, this study examined whether participants experiencing fifteen minutes of mindfulness training would demonstrate greater psychological well-being than the control group, indicated by: greater L > R frontal brain asymmetry and lower SCL during the induction, and greater positive and less negative affect after the emotion induction. Further, this study examined whether participants in the mindfulness group demonstrated greater emotion regulation (and less emotion dysregulation) indicated by: greater HRV during the emotion induction and less selfreported emotion dysregulation and better performance on the AOPSPAN working memory task after the emotion induction. The emotion induction procedure consisted of both pictures (IAPS) and sounds (IADS); participants were randomly assigned to experience either a positive or 
negative induction. Finally, this study sought to determine if emotion regulation mediated the relationship between mindfulness training and psychological well-being.

\section{Mindfulness}

Hypothesis One predicted that a single 15-minute mindfulness training would be sufficient to increase mindfulness as compared to a neutral recording listened to by the control group. Mindfulness was assessed through the self-report of state mindfulness (TMS), and results indicated that Hypothesis One was supported such that: the Mindfulness group reported greater mindfulness than the Control group. To further support the relationship between the brief mindfulness training and increased mindfulness, HRV and frontal brain asymmetry were also assessed at the completion of the 15 minute recording. The Mindfulness group demonstrated greater HRV and L > R frontal brain asymmetry, providing further support for Hypothesis One, and demonstrating that brief mindfulness training increased the subjective and physiological experience of mindfulness.

Hypothesis One also predicted the subjective effects of brief mindfulness training would endure throughout the experiment. This was assessed by comparing the self-report of state mindfulness (TMS) immediately following mindfulness training with state mindfulness measured at the completion of the experimental period. This portion of Hypothesis One was only partially supported such that the mindfulness group reported similar levels of Curiosity (TMS subscale) both immediately after the training and at the termination of the experimental period, but Decentering (TMS subscale), and Total state mindfulness decreased.

Toronto Mindfulness Scale (TMS). Results of the present study extend the findings of Erisman and Roemer (2010) regarding mindfulness training during an experimental period. Erisman and Roemer (2010) developed a 10-minute mindfulness intervention for the purpose of 
producing mindfulness effects in an experimental situation, and utilized the TMS to assess state mindfulness immediately following the intervention and at the completion of the experimental period. The TMS is a state mindfulness measure comprised of two factors, Curiosity and Decentering. The Curiosity subscale is designed to assess the extent to which a participant experienced an increase in awareness colored by openness and curiosity. The Decentering subscale is designed to assess a participant's ability to experience that awareness without becoming entangled with their thoughts and feelings (Lau et al., 2006). Erisman and Roemer (2010) found participants in the Mindfulness condition reported significantly higher levels of Decentering at both time points as compared to the Control condition, but failed to report sufficiently higher Curiosity which resulted in what the authors termed "a modest representation of mindfulness that is better achieved through more extensive practice" (Erisman \& Roemer, 2010, p. 79). Consequently, they suggested that a mindfulness training of only 5 minutes more (15 minutes) would likely increase the state mindfulness reported by participants and instill mindfulness more consistent with clinical applications. The present study adapted Erisman and Roemer's (2010) mindfulness training to extend it to 15 minutes, and results indicated that the 15-minute mindfulness training was sufficient to increase both Decentering and Curiosity as compared to the Control group.

As in Erisman and Roemer (2010), the present study administered the TMS immediately following the intervention and also at the completion of the experimental period to investigate the prolonged effect of the brief intervention. Mindfulness participants' report of Curiosity was maintained throughout the experiment. The Curiosity subscale, representing emotional awareness, appears to be reflected in the increased emotional awareness (greater positive affect regardless of induction and greater negative affect after negative induction) demonstrated by the 
Mindfulness group as compared to the Control group after the emotion induction. Thus, Mindfulness participants' self-report of increased emotional awareness is consistent with their emotional experience after emotional provocation.

Decentering, the TMS subscale representative of the emotion regulation aspect of mindfulness, was reported at significantly lower levels by Mindfulness participants at the end of the experimental period as compared with Decentering immediately following mindfulness training. While this was contrary to the predictions of this study, it appears to be consistent with the emotion regulation results. Mindfulness training failed to create significant differences as compared to the Control group on physiological (HRV) and working memory (AOSPAN) both utilized to assess emotion regulation. Further, mindfulness training resulted in greater self-report of emotion dysregulation (DERS-S) after the negative mood induction. Taken together, these results suggest that the increase in Decentering, a core aspect of emotion regulation, reported immediately following the brief mindfulness training was consumed by efforts to manage emotional experience and was not sufficient to provide improved emotion regulation capacity throughout the study, which is reflected in the emotion regulation measures. Therefore, even though the mindfulness intervention did have a significant effect on the self-report of mindfulness, it had a differential effect on the subjective experience of Curiosity and Decentering after emotional experience which was consistent with the participants' report of emotional awareness (positive and negative PANAS scores) and emotion dysregulation (DERS-S GOALS, IMPULSE, and STATEGIES subscales). Perhaps Decentering, as an emotion regulation skill, needs regular mindfulness practice to be developed (Baer, 2003).

Heart Rate Variability (HRV). In the present study, Mindfulness participants demonstrated greater HRV at the completion of the intervention as compared to Control 
participants. This is consistent with several lines of research (Takahashi et al., 2005; Tang et al., 2009) that have demonstrated autonomic activities during mindfulness training to be characterized by increased parasympathetic activity (higher HRV). Burg, Wolf, and Michalak (2012) suggested this may be due to deep states of relaxation created by mindfulness which would be expected to be associated with higher parasympathetic influence and therefore higher HRV. However, Burg and colleagues (2012) went on to demonstrate that HRV was positively correlated with mindfulness as measured by the mindful breathing exercise (MBE; Burg \& Michalak, 2011), which assessed mindfully staying in contact with the breath through selfregulated attention during a breathing meditation exercise. This finding suggests that the relationship between mindfulness training and higher HRV is not due merely to relaxation, but to self-regulated attention to the present moment, a central mindfulness practice (Burg, Wolf, \& Michalak, 2012). In addition, no significant differences in HRV were demonstrated between groups prior to the intervention. Taken together, the present findings suggest that the higher HRV of Mindfulness participants at the completion of mindfulness training is indicative of what Mankus and colleagues (2013) agree is an increase in the flexible emotional responding that is characteristic of mindfulness.

Frontal Brain Asymmetry (EEG laterality coefficients). Previous research (e.g., Keune et al., 2013; Moyer et al., 2011) suggests that L > R frontal brain activation is a pattern indicative of approach-motivation (as opposed to avoidance or withdrawal) and the capacity for emotion regulation characteristic of mindfulness. This is consistent with several lines of research which collected EEG data during mindfulness training and have found that $\mathrm{L}>\mathrm{R}$ frontal brain activation as a result of mindfulness training is indicative of stronger approach tendencies (Barnhofer, Chittka, Nightingale, Visser, \& Crane, 2010), the ability to recover quickly from 
negative emotional experience (Keune et al., 2013), and curiosity about new experiences

(Takahashi et al., 2005). The findings of this study demonstrate that 15 minutes of mindfulness training is sufficient to shift frontal brain activation to this pattern, such that the mindfulness group experienced greater $\mathrm{L}>\mathrm{R}$ frontal brain asymmetry at the completion of the mindfulness training as compared to the control group. Keune and colleagues (2013) suggest the L > R frontal brain asymmetry is indicative of an approach-oriented emotional state, and Moyer et al. (2011) agrees, suggesting this pattern indicates a willingness to move toward emotional experience rather than to avoid or escape. Therefore, these results suggest that a 15 -minute mindfulness training is sufficient to not only increase emotional awareness but also to reduce avoidance of emotional experience. This is consistent with the self-report of positive and negative affect described below, which suggests that participants in the mindfulness condition reported greater contact with and awareness of both their positive and negative emotional experience than the Control condition.

\section{Emotional Well-being}

Hypothesis Two predicted that mindfulness training would improve emotional wellbeing, assessed by self-report of positive and negative affect after an emotion induction (Positive or Negative) as well as by neurological (frontal brain asymmetry) and physiological (SCL) measures during the emotion induction. This hypothesis was partially supported in that: positive affect was higher in the Mindfulness group as compared to the control, however, negative affect was not lower for the same; frontal brain asymmetry was in the predicted direction (Mindfulness group demonstrating $\mathrm{L}>\mathrm{R}$ frontal brain activation as compared to the Control group), but did not achieve significance; no significant differences were demonstrated between groups on SCL. 
Positive and Negative Affect (PANAS). The findings of this study suggest that participants receiving 15 minutes of mindfulness training experienced greater emotional awareness during the emotion induction, indicated by greater self-reported positive affect regardless of induction (positive or negative) and greater negative affect when experiencing a negative induction. These results were contrary to the prediction that mindfulness training would result in greater positive and lower negative affect after emotional provocation. Despite this prediction, the present findings suggest the effectiveness of the brief mindfulness training to increase awareness and reduce avoidance of emotional experience.

A number of studies have demonstrated a positive correlation between length of mindfulness training and its effects on various physiological, neurological and behavioral measures, which may suggest the brevity of the mindfulness training resulted in the differential impact on positive and negative affect. For example, Grant and colleagues (2010) demonstrated that increases in cortical thickness in several different brain regions had a positive correlation with the length of mindfulness training. However, Jha et al. (2010) found differential effects of mindfulness training on positive and negative affect as a result of greater mindfulness practice effects on improvements in working memory. Specifically, they found working memory capacity (AOSPAN scores), which corresponds to the ability to successfully regulate emotion, was greater in participants with higher mindfulness practice time during a stressful eight week period as compared to those with lower practice time. In addition, Jha et al. (2010) found differential effects of mindfulness training ( 8 weeks) on positive and negative affect (PANAS positive and negative scores) as a result of improvement in working memory. Their findings suggested that there was a direct effect of mindfulness training in increasing positive affect. However, the effects of mindfulness training in lowering negative affect were mediated by working memory 
capacity (AOPSAN scores). Jha et al. (2010) suggested that increased working memory capacity, while being unrelated to positive affect, benefited the regulation of negative affect because only the experience of negative affect requires regulation. Therefore, the relationship between length of mindfulness training and emotion regulation capacity may account for the unexpected effect of the present study's brief mindfulness training on negative affect as compared to positive affect. It appears that the direct effects of the brief mindfulness training, which increased awareness and reduced avoidance of emotional experience (positive and negative), were sufficient to improve the participants' experience of positive emotion. However, one brief mindfulness training did not sufficiently improve emotion regulation that may have mediated the relationship between mindfulness and reduced distress when experiencing negative affect (Jha et al., 2010). Therefore, it is hypothesized that continued mindfulness practice would be necessary to improve the participants' reaction to negative affect during the experience of unpleasant stimuli (e.g., negative emotion provocation) as has been found by Jha et al. (2010) and Davidson (2010).

Frontal Brain Asymmetry (EEG laterality coefficients). Previous research (Keune et al., 2013; Moyer et al., 2011) suggests that L > R frontal brain activation is a pattern indicative of approach-motivation and the capacity for emotion regulation characteristic of mindfulness. In the current study, frontal brain asymmetry was in the predicted direction during the emotion induction, such that Mindfulness participants demonstrated L > R frontal brain asymmetry (EEG laterality coefficient $>1$ ) and Control participants did not (EEG laterality coefficients $<1)$; however, the difference between groups did not reach significance. Assessing frontal brain asymmetry during the emotion induction was originally intended to measure the experience of emotion (Davidson et al., 1990) as an indicator of psychological well-being. However, if we 
follow the thinking of some previous research (e.g., Jackson et al., 2003) suggesting frontal brain asymmetry reflects the role of the prefrontal cortex in emotion regulation then the failure to demonstrate significant differences in EEG laterality coefficients between groups during the emotion induction is consistent with the lack of significant differences on other measures of emotion regulation (see Emotion Regulation below).

Jackson and colleagues (2003) provide support for the relationship between the present frontal brain asymmetry findings and the differential effects of mindfulness training on emotional experience and emotion regulation. They examined frontal brain asymmetry in participants who viewed arousing and neutral pictures (IAPS) while also collecting eye-blink startle data. Participants demonstrating greater L > R frontal activation also demonstrated shorter duration of negative affect (less eye-blink startle magnitude) after emotional provocation. Further, L > R frontal brain activation solely predicted post-picture emotional recovery and not initial emotional reactivity, suggesting this pattern of neural responding is more closely related to emotion regulation than emotional experience (Jackson et al., 2003).

Similarly, Harmon-Jones and colleagues (2003) found L > R frontal brain activation occurred in response to an anger provocation only when participants believed coping responses would be possible. In their study, college students heard either a recording that stated tuition would definitely be increasing or one stating that a tuition increase was being considered. Participants led to believe the increase was only under consideration were more likely to engage in coping actions (e.g., signing petitions) and demonstrated greater $\mathrm{L}>\mathrm{R}$ frontal brain activation than those who believed the increase was unavoidable. In other words, when participants thought they would be able to do something about the anger provoking situation, they responded with $\mathrm{L}$ $>\mathrm{R}$ frontal brain activation (Harmon-Jones et al., 2003). The authors suggest that this not only 
supports the approach/withdrawal literature indicating that $\mathrm{L}>\mathrm{R}$ frontal brain asymmetry represents approach motivation as opposed to avoidance of experience or withdrawal (Keune et al., 2013; Sobotka, Davidson, \& Senulis, 1992), but also supports L > R frontal brain asymmetry as something that facilitates and regulates emotional responding (Harmon-Jones et al., 2003).

The 15-minute mindfulness training of the present study increased approach-motivation for emotional experience and the capacity for emotion regulation characteristic of mindfulness. This was indicated by greater relative left-frontal brain activity at the completion of the intervention as well as greater Curiosity and Decentering (TMS subscale) reported immediately following the intervention as compared to the Control group. However, mindfulness participants failed to maintain the increase in L > R frontal brain activity and Decentering under emotional provocation. Mindfulness training encourages an openness to experience without attempts to change (Curiosity) as well as the ability of an individual to have that experience without becoming entangled in their thoughts and feelings (Decentering). These results suggest that $\mathrm{L}>$ $\mathrm{R}$ frontal brain activation may have a stronger relationship with Decentering (objective perspective on the experience of emotion) than with Curiosity (emotional awareness and reduced avoidance). Furthermore, these results suggest that 15-minutes of mindfulness training reduces avoidance of emotional experience and improves emotion regulation capacity, but not sufficiently to surmount the demands of managing emotional experience. Since there is significant evidence that mindfulness training effects, including emotion regulation, increase with length of mindfulness training (Jha et al., 2010; Davidson, 2010), and given that greater relative left-frontal brain activity was in the predicted direction during the emotion induction, it is suggested that $\mathrm{L}>\mathrm{R}$ frontal brain asymmetry would be maintained despite emotional experience as mindfulness practice continued. 
Skin Conductance Level (SCL). The present study did not find any significant differences in SCL for participants in the mindfulness group as compared to the control group during the emotion induction. While failing to support the prediction of Hypothesis Two, these results are consistent with several studies which utilized SCL as an indicator of emotional experience in response to mindfulness. For instance, Levitt, Brown, Orsillo, and Barlow (2004) found that acceptance, an aspect of mindfulness, resulted in less subjective anxiety and avoidance as compared to a group asked to suppress their emotions, but found no differences between conditions in SCL. The authors suggested that acceptance increased participants' willingness to experience emotion without altering their physiological experience of those emotions. Several studies (Eifert \& Heffner, 2003; Vernig \& Orsillo, 2009; Erisman \& Roemer, 2010) found similar results, suggesting that mindfulness may be impacting distress about emotional experience rather than emotional responding itself. This differential emotional responding is explained by Lang's tripartite model, which suggests that emotion, especially fear, is comprised of three separate but related components: physiological arousal, cognitive (subjective) distress, and behavioral avoidance (Ollendick, Allen, Benoit, \& Cowart, 2011). This model states that the components may co-vary, but are capable of responding independently. Therefore, an individual may experience high subjective distress and neither high physiological arousal nor avoidance of the experience, which is consistent with the current findings. Furthermore, the results of the present study support the assertion that mindfulness training effects on the subjective experience of emotion are distinct from its effects on sympathetic arousal (SCL), perhaps impacting distress about emotional experience rather than emotional responding itself (Vernig \& Orsillo, 2009). 


\section{Emotion Regulation}

Hypothesis Three predicted that mindfulness training would demonstrate a significant increase in emotion regulation and decrease in emotion dysregulation as compared to the Control condition. This was assessed with self-report of emotion dysregulation (DERS-S), HRV and performance on a working memory task (AOSPAN). This prediction was not supported, in that: there was no main effect of Condition (Mindfulness or Control) on any of the three outcome variables.

State Difficulties in Emotion Regulation Scale (DERS-S). Examination of the selfreport of emotion dysregulation revealed a significant interaction such that participants in the mindfulness condition who experienced the negative emotion induction reported greater emotion dysregulation than any other condition (i.e., Mindfulness Positive, Control Positive, Control Negative). Specifically, these participants reported greater difficulties engaging in goal directed behavior (GOALS; reflects difficulty concentrating when experiencing emotions) and endorsed items regarding their emotions such as, "I have difficulty thinking about anything else," and "I have difficulty concentrating". They also indicated impulse control difficulties (IMPULSE; reflects difficulty having a sense of control when experiencing emotions), endorsing items such as "I experience my emotions as overwhelming and out of control," and "I feel out of control". Finally, they reported limited access to emotion regulation strategies (STRATEGIES; reflects the belief that there is little one can do to alter their emotions and they will persist), endorsing items regarding their emotions such as "I believe that there is nothing I can do to make myself feel better," and "My emotions feel overwhelming". Therefore, participants in the mindfulness condition who experienced the negative mood induction reported feeling more overwhelmed, out of control, and unable to improve their emotional state. 
This interaction in which participants in the mindfulness condition experiencing the negative induction reported feeling more overwhelmed and unable to improve their emotional state coincides with this same group reporting significantly greater negative affect. Considering mindfulness training effects on positive and negative affect, results suggest that participants in the Mindfulness condition responded to the mindfulness training as instructed (not attempting to reduce or control negative emotion) which resulted in greater experience of both positive (given either positive or negative inductions) and negative (given negative induction) affect. Thus, it appears that openness to experience caused by mindfulness training made the negative mood induction more intense resulting in the subjective experience of feeling more dysregulated.

In addition, analysis of the role of emotion dysregulation in the relationship between mindfulness training and psychological well-being provided further support for the positive correlation between emotion dysregulation and negative affect. Specifically, greater difficulty concentrating when experiencing emotions (GOALS), feeling helpless to alter one's emotions (STRATEGIES), and general emotion dysregulation (DERS-S total score) correlated with greater negative affect. This is consistent not only with the interactions described above, but also with previous research suggesting greater emotion dysregulation is positively correlated with greater negative affect (Vujanovic et al., 2010; Sears \& Kraus, 2009; Ehring et al., 2010).

Interestingly, the greater report of emotion dysregulation by mindfulness participants after the negative emotional experience provides further support for the effectiveness of the brief mindfulness training. Erisman and Roemer (2010) failed to demonstrate a significant effect of their 10-minute mindfulness training on both the self-reported emotion dysregulation (DERS-S) and self-reported state mindfulness (TMS total score). The 15-minute mindfulness training of the present study resulted in significant increases in mindfulness compared to the Control condition, 
and, when followed by a negative emotional experience, resulted in greater self-report of negative affect and emotion dysregulation. This contrast in results provides further support for the assertion that 15 -minutes of mindfulness training is sufficient to increase emotional awareness which appears to have increased the experience of emotions being overwhelming and out of control.

It seems that, as with the frontal brain asymmetry results, the findings regarding the selfreport of Curiosity, the TMS subscale representative of emotional awareness, and Decentering, the TMS subscale representative of the emotion regulation aspect of mindfulness, can help explain the findings regarding greater emotion dysregulation being reported by Mindfulness participants after a negative mood induction. Greater Curiosity was maintained throughout the experimental session, which is reflected in Mindfulness participants' greater report of emotional awareness (positive and negative PANAS scores), particularly the greater awareness of negative emotion after a negative emotional experience. Greater Decentering reported immediately following the brief mindfulness training was consumed by efforts to manage emotional experience and was not sufficient to provide improved emotion regulation capacity for the duration of the experimental period. This is reflected in Mindfulness participants' report of emotion dysregulation (DERS-S GOALS, IMPULSE, and STATEGIES subscales). Therefore, the differential effect a 15-minute mindfulness training on the subjective experience of Curiosity and Decentering after emotional experience is not only consistent with the participants' report of emotional awareness and emotion dysregulation, but offers an explanation for the same. Specifically, mindfulness training increased emotional awareness which increased the experience of negative affect after negative emotion provocation without sufficiently improving emotion regulation to manage this more intense emotional experience. 
The findings of the present study regarding emotion regulation have significant implications for the effective treatment of psychological disorders with mindfulness-based interventions. Emotion dysregulation has been associated with a variety of forms of psychopathology, including anxiety (Olatunji, Forsyth, \& Feldner, 2007), depression (Ehring et al., 2010), anger and aggression (Pond, Kashdan, DeWall, Savostyanova, Lambert, \& Fincham, 2012) and decline in interpersonal relationship quality (Smith et al., 2011). As such, much mindfulness research continues to explore ways to improve emotion regulation through mindfulness-based interventions (e.g., Davidson et al., 2003; Keune et al., 2013; Shapiro et al., 2007), and several have been determined to successfully use mindfulness training as a path to improved emotion regulation. For example, mindfulness-based cognitive therapy (MBCT; Teasdale et al., 2000) and dialectical behavior therapy (DBT; Lynch et al., 2006) employ mindfulness training to address "ineffective action tendencies linked with dysregulated emotion" (p. 459). In both of these cases, mindfulness is employed, not to change emotional experience, but to increase the patient's ability to respond adaptively to their emotional experience, ergo improved emotion regulation.

However, the results of the current study suggest that the initial experience of increased mindfulness may be difficult for people, especially when experiencing negative emotion, which has important implications for the clinical application of mindfulness training. For example, these results indicate the timing of initial mindfulness training (e.g., not when the patient is experiencing intense distress) should be taken into consideration. In addition, this indicates a need for providing psychoeducation regarding the possibility that a patient may experience their emotions as more overwhelming when mindfulness training begins. Furthermore, results indicate that a single mindfulness training may not increase emotion regulation ability sufficiently to 
reduce the subjective experience of emotion dysregulation after a negative experience.

Therefore, in an effort to prepare the patient to manage the increase in emotional awareness they will experience, perhaps training in emotion regulation skills (as in DBT; Lynch et al., 2006) could be implemented before the onset of mindfulness training. Research suggests that length of mindfulness training demonstrates a positive relationship with greater mindfulness skills in general (Grant et al., 2010), and emotion regulation characteristic of mindfulness specifically (Jha et al., 2010); therefore the initial experience of increased mindfulness as difficult and overwhelming would be mitigated with continued mindfulness practice.

Heart Rate Variability (HRV). The measure of HRV, the coupling of heart rate and respiration, provides a reliable measure of parasympathetic nervous system activation (Berntson et al., 1997), has been shown to underlie crucial aspects of emotion regulation (Thayer, Ahs, Fredrikson, Sollers, \& Wager, 2012; Thayer \& Lane, 2009), and has been employed in many experimental studies as an indicator of emotional response to emotional experience (e.g., Burg, Wolf, \& Michalak, 2012; Mankus et al., 2013; Smith et al., 2011). Greater changes in heart rate across the respiratory cycle (i.e., increases in HRV) indicate greater parasympathetic activation, which is evoked by efforts to regulate emotion and behavior (Smith et al., 2011).

As previously stated, mindfulness training resulted in significantly greater HRV at the completion of the intervention. Considering there were no significant differences between groups on HRV prior to mindfulness training, the increase in HRV for Mindfulness participants is most certainly due to mindfulness training. Furthermore, previous research indicates that: autonomic activities during mindfulness training are characterized by higher HRV (Takahashi et al., 2005); and are not due merely to relaxation, but to self-regulated attention to the present moment, a central mindfulness practice (Burg, Wolf, \& Michalak, 2012). Therefore, these findings suggests 
that the higher HRV at the completion of mindfulness training in the present study is an indication of increased mindfulness.

In contrast to predictions, HRV was not found to be significantly different between groups during the emotion induction. While HRV was greater for the Mindfulness group after the intervention, it remained essentially unchanged during the emotion induction (positive or negative). While this does not support the prediction of Hypothesis Three regarding HRV being greater due to mindfulness training as compared to Controls, the findings of Burg, Wolf, and Michalak (2012) suggest the HRV differences demonstrated between groups at the completion of the intervention may still reflect a self-regulatory state. These authors describe mindfulness as "striving to stay in contact with the experience of the present moment in an attentive, conscious and accepting manner," stating that "Mindfulness is therefore highly characterized by selfregulatory effort" (p. 136). Their findings indicated that participants better able to self-regulate their attention to their breathing (mindfulness exercise) displayed significantly higher HRV (Burg et al., 2012). They asserted, with HRV as an indicator of the ability to regulate emotions and self-regulation as a core aspect of mindfulness, that HRV is an important correlate of the self-regulatory nature of mindfulness. Considering this, it could be asserted that the greater HRV demonstrated by the Mindfulness group at the completion of the intervention represents an increase in mindfulness; furthermore, the lack of change in HRV for this group during the emotion induction suggests that mindfulness participants maintained that more mindful state despite emotion provocation. Thus, while the HRV data was not as predicted, these results still demonstrate the effects of mindfulness training on increasing and maintaining self-regulatory effort during an emotion induction. 
Additional support exists for this assertion. First, Mindfulness participants were given specific instructions immediately before the emotion induction, stating, "If you notice any emotions during the presentation of pictures and sounds, try to just acknowledge and accept them as they are, without trying to change your experience in any way." Thus, the self-regulatory effort of Mindfulness participants indicated by the maintenance of increased HRV during the emotion induction was likely due to following these instructions, suggesting the attempt to utilize mindfulness. Second, the maintenance of HRV increases during the emotion induction by Mindfulness participants occur in conjunction with; 1) the maintenance of Curiosity (TMS subscale of emotional awareness and reduced avoidance); and greater self-report of emotional awareness (greater PANAS positive score regardless of induction and greater PANAS negative scores after negative emotion induction). Considering this, the maintenance of increased HRV of Mindfulness participants during the emotion induction could be related to mindfulness effects of increased emotional awareness and reduced avoidance of emotional experience. Taken together, it seems likely that the increased HRV of Mindfulness participants maintained during emotional provocation represents the continued self-regulatory efforts characteristic of mindfulness.

While this interpretation seems meaningful, the failure of mindfulness training to result in significantly greater HRV during the emotion induction as compared to the Control group may suggest that 15-minutes of mindfulness training reduces avoidance of emotional experience and improves emotion regulation capacity, but not sufficiently to surmount the demands of managing emotional experience. If HRV is an indicator of the flexibility of the autonomic nervous system to respond adaptively to emotional demands for regulation, as with all other emotion regulation findings of this study, it is predicted that significant differences would emerge between groups during emotion induction with greater mindfulness practice (e.g., Grant et al., 2010). 
Another explanation for the lack of significant differences in HRV during the emotion induction despite mindfulness training is offered by Mankus, Aldao, Kerns, Mayville, and Mennin (2013), who examined the relationship between trait mindfulness, generalized anxiety symptoms, and HRV. They discovered a significant interaction such that participants demonstrating low anxiety displayed no significant relationship between mindfulness and HRV. However, for those in the high anxiety group, mindfulness scores demonstrated a positive correlation with HRV. As higher anxiety is associated with increased emotion dysregulation, Mankus et al. (2013) suggest these findings indicate mindfulness is an adaptive strategy that predicts parasympathetic influence (HRV) with individuals who have a greater tendency to employ maladaptive strategies (i.e., greater emotion dysregulation). Trait emotion dysregulation scores reported at the onset of the current study indicated no significant differences between conditions regarding maladaptive emotion regulation. Furthermore, these scores for both the Mindfulness and Control participants $(M=74.24, S D=18.84)$ are slightly lower than the average means $(M=79.33, S D=19.72)$ demonstrated by participants in the initial study to validate the Dysregulation in Emotion Regulation Scale (DERS; Gratz \& Roemer, 2004). Therefore, there may have been no significant differences between conditions regarding HRV during the emotion induction since the groups weren't different in their tendency to experience emotion dysregulation.

Working Memory Capacity (AOSPAN). The present study examined the ability of a brief mindfulness training to create greater working memory capacity (WMC; a correlate to the ability to successfully regulate emotion; Jha et al., 2010) as compared to the Control group. No significant effects were revealed, and therefore Hypothesis Three was not supported. As previously discussed regarding self-reported negative affect and emotion dysregulation, prior 
research suggests that the length of mindfulness training has a positive relationship with mindfulness skills (Grant et al., 2010) and more specifically, improvements in WMC (Jha et al., 2010). It is therefore likely that a single, brief mindfulness intervention was not sufficient to create improvements in working memory capacity consistent with previous research that has used much longer interventions (e.g., 8 weeks; Jha et al., 2010). Furthermore, this author is not aware of any studies with a single session of mindfulness training that resulted in improvements in WMC.

Other possibilities for these nonsignificant results regarding WMC are indicated by previous research. For example, Goodman and colleagues (2013) indicated that differences in working memory between participants demonstrating $\mathrm{L}>\mathrm{R}$ frontal brain activation emerged only under conditions of sufficient stress, suggesting that the pictures and sounds utilized in the present study, while sufficient to induce both positive and negative emotion, may not have induced sufficient stress to reveal differences in performance on the working memory task. However, Deveney and Pizzagalli's (2008) research seems more consistent with the complete picture painted by the present findings. Their results suggest that any improvements mindfulness training may have made in WMC in the present study were consumed during the emotion induction prior to performance on the AOSPAN. Deveney and Pizzagalli (2008) had participants regulate emotions to unpleasant pictures and then complete a cognitive task to examine the cognitive consequences of emotion regulation. Their findings suggest that regulating affect, during the viewing of unpleasant stimuli, might consume cognitive resources and continue to impact resources available to process information after the emotional experience has passed. Regarding the present study, this suggests the lack of significant differences in WMC could be 
the result of emotion regulation consuming cognitive resources during the emotion induction as opposed to the assumption that mindfulness training had no impact on WMC.

\section{Mindfulness Training, Emotion Regulation, and Psychological Well-being}

The mediational analyses failed to reveal the mediation of mindfulness training effects on psychological well-being by emotion regulation. The Preacher and Hayes (2008) mediational model utilized in the present study indicates that indirect effects can be present when total effects are absent; therefore, even if the independent variable fails to demonstrate change in the dependent variable, mediation is still possible (Hayes, 2009). Despite this, it seems likely that the lack of significant effects of mindfulness training on two of the three emotion regulation variables (HRV and WMC) as well as unexpected results regarding the third emotion regulation variable (DERS-S subscales and total), must have an impact on these mediational results. For example, WMC mediated the relationship between mindfulness training and negative affect in Jha et al. (2010), but as previously discussed, it may be that the present study's single session mindfulness intervention was insufficient to significantly improve WMC. The interaction in which mindfulness training followed by a negative emotion induction resulted in the report of greater negative affect and greater emotion dysregulation further suggests that the brief training was sufficient to increase emotional awareness, but perhaps not sufficient to improve selfreported regulation of those emotions. Therefore, it is possible that the failure of mediational analysis to reveal a mediational role of emotion regulation (HRV, WMC, and DERS-S) in the relationship between mindfulness training and psychological well-being (PANAS positive and negative scores, frontal brain asymmetry, and SCL), is not an accurate depiction of emotion regulation's role, but a reflection of the above discussed limitations of the brief mindfulness training. It is likely, based on the results of this study and previous research in emotion 
regulation (Jha et al., 2010; Deveney \& Pizzagalli, 2008; Mankus et al., 2013), that increased length of mindfulness training may be required to illustrate a mediational relationship with emotion regulation and psychological well-being.

\section{Strengths}

This study boasts several strengths. First, self-report, physiological, and neurological measures were used to assess potentially differential effects of mindfulness training on an individual's subjective experience of emotion (e.g., report of more positive affect after mindfulness training) and their physiological arousal (e.g., no significant differences in SCL during the induction) and neurological responding (e.g., EEG laterality coefficient moved in the predicted direction but did not achieve significance during the emotion induction). Second, this study examined positive as well as negative psychological outcomes in consideration of the possibility that mindfulness training would impact these outcomes differentially (Jha et al., 2010). Third, the mindfulness intervention was presented as a recording to insure consistency of the training throughout the study and to limit the potential for participant response due to social desirability factors that may occur when training is done in person. Fourth, pictures (IAPS) and sounds (IADS) standardized on the basis of normative ratings with respect to valence and arousal were used in the emotion induction to insure: a) the induction was sufficiently emotionally evocative; and b) differences in emotional responding to the positive and negative induction would be due to mindfulness effects and not the negative condition being experienced as more arousing. Fifth, performance on a working memory task (AOSPAN) was used as a non-selfreport indicator of emotion regulation capacity. Finally, to the best of our knowledge, this study represents the first attempt to examine the effects of a single mindfulness training session on 
emotion regulation, and has suggested important considerations for its application to clinical settings.

\section{Limitations and Future Directions}

First, the current findings were based on a relatively homogeneous, non-clinical sample of young adults in college. It is important for future research to examine the relationship between brief mindfulness training, emotion regulation, and psychological well-being in a population experiencing clinical levels of emotion dysregulation to enable us to answer questions regarding specific disorders. Further, it would be useful to examine mindfulness training, emotion regulation, and psychological well-being in a more developmentally diverse population or one with more varied backgrounds. Second, the electrodes placed on the scalp were reported as mildly to very uncomfortable by many participants by the end of the 1.5 hour experimental period. This increased discomfort could have engendered feeling of distress beyond that intended by the emotion induction, which may have affected outcomes. Third, the AOSPAN, of a twenty minutes duration completed at the end of the experimental period, was often reported as aversive, including references to hating math and feeling anxiety or fatigue. It may be useful in future studies of emotion regulation to utilize cognitive tests that eschew math problems or are briefer in nature. Finally, the brief mindfulness training reduced avoidance of emotional experience and improved emotion regulation capacity, but not sufficiently to surmount the demands of managing emotional experience, which we believe accounts for the lack of significant mediational results. As such, the present findings are proposed to be an underestimate of the results possible with a mindfulness practice of longer duration. Clearly, future studies employing mindfulness training of a longer duration should continue to examine the role of emotion regulation in the relationship 
between mindfulness training and psychological well-being to better illuminate the mechanisms of mindfulness.

\section{Conclusions}

This present study employed physiological measures and a working memory task in addition to self-report measures to seek a more accurate understanding of the relationship between brief mindfulness training and the experience and regulation of emotion. Results indicated that the brief mindfulness training was sufficient to increase mindfulness, demonstrated by significantly greater: self-report of mindfulness, L $>$ R frontal brain activation, and HRV. In addition, participants receiving 15-minutes of mindfulness training experienced greater emotional awareness during the emotion induction, indicated by reporting greater positive affect regardless of type of induction they received and greater negative affect when experiencing a negative induction. Therefore, brief mindfulness training is sufficient to not only increase emotional awareness but to also create changes in brain activity and parasympathetic activation indicative of the ability to respond adaptively to emotional experience.

However, the results of the current study suggest that the initial experience of increased mindfulness may be difficult for people, especially when experiencing negative emotion, since experiencing a negative emotion induction after mindfulness training also resulted in participants feeling more overwhelmed and unable to improve their emotional state. We hypothesize that openness to experience caused by mindfulness training made the negative mood induction more intense resulting in the subjective experience of feeling more dysregulated. In addition, while Mindfulness participants demonstrated $\mathrm{L}>\mathrm{R}$ frontal brain activity during the emotion induction, and maintained increases in HRV resulting from mindfulness training under emotional provocation, differences did not reach significance. These results suggest that 15 -minutes of 
mindfulness training reduces avoidance of emotional experience and improves emotion regulation capacity, but not sufficiently to surmount the demands of managing emotional experience. Also, the lack of significant differences between conditions regarding performance on the working memory task is hypothesized to be due to the brief nature of the mindfulness training employed. Since there is significant evidence that the effects of mindfulness training, including emotion regulation, increase with length of mindfulness training (Jha et al., 2010; Davidson, 2010), it is suggested that the self-report of emotion regulation difficulties would decrease and $\mathrm{L}>\mathrm{R}$ frontal brain asymmetry, $\mathrm{HRV}$, and WMC would be greater despite emotional experience as mindfulness practice continued. Finally, there are significant clinical implications for the initial experience of increased mindfulness as difficult when experiencing a negative emotion. 


\section{References}

Batchelor, S. (1994). The awakening of the West: The encounter of Buddhism and Western culture. Berkeley, CA: Parallel Press.

Baer, R.A. (2003). Mindfulness training as a clinical intervention: A conceptual and empirical review. Clinical Psychology: Science and Practice, 10, 125-143.

Baer, R.A., Smith, G.T., \& Allen, K.B. (2004). Assessment of mindfulness by self report: The Kentucky Inventory of Mindfulness Skills. Assessment, 11, 191-203.

Barnhofer, T., Chittka, T., Nightingale, H., Visser, C., \& Crane, C. (2010). State effects of two forms of meditation on prefrontal EEG asymmetry in previously depressed individuals. Mindfulness, 1, 21-27.

Berntson, G.G., Bigger, J.T., Eckberg, D.L., Grossman, P., Kaufmann, P.G., Malik, M.,... van der Molen, M.W. (1997). Heart rate variability: Origins, methods, and interpretive caveats. Psychophysiology, 34, 623-648.

Bradley, M. M., \& Lang, P. J. (1999). International affective digitized sounds (IADS): Stimuli, instruction manual and affective ratings (Tech. Rep. No. B-2). Gainesville, FL: The Center for Research in Psychophysiology, University of Florida

Brown, K.W. \& Ryan, R.M. (2003). The benefits of being present: Mindfulness and its role in psychological well-being. Journal of Personality and Social Psychology, 84, 822-848.

Burg, J.M., \& Michalak, J. (2011). The healthy quality of mindful breathing: Associations with rumination and depression. Cognitive Therapy and Research, 35, 179-185.

Burg, J.M., Wolf, O.T., Michalak, J. (2012). Mindfulness as self-regulated attention: Associations with heart rate variability. Swiss Journal of Psychology, 71, 3, 135-139. 
Campbell-Sills, L., Barlow, D. H., Brown, T. A., \& Hofmann, S. G. (2006). Effects of suppression and acceptance on emotional responses on individuals with anxiety and mood disorders. Behavior Research and Therapy, 44, 1251-1263.

Carlson, L.E. \& Brown, K.W. (2005). Validation of the Mindful Attention Awareness Scale in a cancer population. Journal of Psychosomatic Research, 58, 29-33.

Coffey, K.A., Hartman, M., \& Fredrickson, B.L. (2010). Deconstructing mindfulness and constructing mental health: Understanding mindfulness and its mechanisms of action. Mindfulness, 1, 235-253.

Colden, A., Bruder, M., \& Manstead, A.S.R. (2008). Human content in affect-inducing stimuli: A secondary analysis of the international affective picture system. Motivation and Emotion, 32, 260-269.

Conway, A.R.A., Cowan, N., \& Bunting, M.F. (2001). The cocktail party phenomenon revisited: The importance of working memory capacity. Psychonomic Bulletin and Review, 8, 331335.

Davidson, R. J., Ekman, P., Saron, C. D., Senulis, J., \& Friesen, W. (1990). Approach/withdrawal and cerebral asymmetry: Emotional expression and brain physiology I. Journal of Personality and Social Psychology, 58, 330-341.

Davidson, R. J., \& Fox, N. A. (1989). Frontal brain asymmetry predicts infants' response to maternal separation. Journal of Abnormal Psychology, 9, 127-131.

Davidson, R.J. (2000). Affective style, psychopathology, and resilience: Brain mechanisms and plasticity. American Psychologist, 55, 1196-1214.

Davidson, R.J. (2010). Empirical explorations of mindfulness: Conceptual and methodological conundrums. Emotion, 10, 1, 8-11. 
Deveney, C.M., \& Pizzagalli, D.A. (2008). The cognitive consequences of emotion regulation: An ERP investigation. Psychophysiology, 45, 435-444.

Ehring, T., Tuschen-Caffier, B., Schnulle, J., Fischer, S., and Gross, J.J. (2010). Emotion regulation and vulnerability to depression: Spontaneous versus instructed use of emotion suppression and reappraisal. Emotion, 10, 563-572.

Eifert, G.H., \& Heffner, M. (2003). The effects of acceptance versus control contexts on avoidance of panic-related symptoms. Journal of Behavior Therapy and Experimental Psychiatry, 34, 293-312.

Erisman, S.M., \& Roemer, L. (2010). A preliminary investigation of the effects of experimentally induced mindfulness on emotional responding to film clips. Emotion, 10, 72-82.

Farb, N.A.S., Anderson, A.K., Mayberg, H., Bean, J., McKeon, D., \& Segal, Z.V. (2010). Minding one's emotions: Mindfulness training alters the neural expression of sadness. Emotion, 10, 25-33.

Field, A. (2013). Discovering Statistics Using IBM SPSS Statistics. Los Angeles: Sage Publications.

Frewen, P.A., Evans, E., Maraj, N., Dozois, D.J.A., \& Partridge, K. (2007). Letting go: Mindfulness and negative automatic thinking. Cognitive Therapy and Research, 32, 758774.

Goldin, P.R., \& Gross, J.J. (2010). Effects of Mindfulness-Based Stress Reduction (MBSR) on emotion regulation in social anxiety disorder. Emotion, 10, 83-91.

Goodman, R.N., Rietschel, J.C., Lo, L., Costanzo, M.E., \& Hatfield, B.D. (2013). Stress, emotion regulation and cognitive performance: The predictive contributions of trait and 
state relative frontal EEG alpha asymmetry. International Journal of Psychophysiology, $87,115-123$.

Grant, J.A., Courtemanche, J., Duerden, E.G., Duncan, G., \& Rainville, P. (2010). Cortical thickness and pain sensitivity in Zen meditators. Emotion, 10, 43-53.

Gratz, K.L., \& Roemer, L. (2004). Multidimensional assessment of emotion regulation and dysregulation: Development, factor structure, and initial validation of the Difficulties in Emotion Regulation Scale. Journal of Psychopathology and Behavioral Assessment, 26, 41-54.

Gross, J.J. (1998) Antecedent- and response-focused emotion regulation: Divergent consequences for experience, expression, and physiology. Journal of Personality and Social Psychology, 74, 224-237.

Gross, J.J. (2002). Emotion regulation: Affective, cognitive, and social consequences. Psychophysiology, 39, 281-291.

Gross, J.J., \& Thompson, R.A. (2007). Emotion regulation: Conceptual foundations. In J.J. Gross (Ed.), Handbook of Emotion Regulation (p. 3-26). New York: Guilford Press.

Harmon-Jones, E., Sigelman, J.D., Bohlig, A., \& Harmon-Jones, C. (2003). Anger, coping, and frontal cortical activity: The effect of coping potential on anger-induced left frontal activity. Cognition and Emotion, 17, 1-24.

Hayes, A.F. (2009). Beyond Baron and Kenny: Statistical mediation analysis in the new millennium. Communication Monographs, 76, 408-420.

Hayes, A.M., \& Feldman, G. (2004). Clarifying the construct of mindfulness in the context of emotion regulation and the process of change in therapy. Clinical Psychology: Science and Practice, 11, 255-262. 
Hill, C.L., \& Updegraff, J.A. (2012). Mindfulness and its relationship to emotion regulation. Emotion, 12, 81-90.

Jackson, D.C., Mueller, C.J., Dolski, I., Dalton, K.M., Nitschke, J.B., Urry, H.L., Rosenkranz, M.A., Ryff, C.D., Singer, B.H., \& Davidson, R.J. (2003). Now you feel it, now you don't: Frontal Brain Electrical Asymmetry and Individual differences in emotion regulation. Psychological Science, 14, 6, 612-617.

Jha, A.P., Stanley, E.A., Kiyonaga, A., Wong, L., \& Gefland, L. (2010). Examining the protective effects of mindfulness training on working memory capacity and affective experience. Emotion, 10, 54-64.

Kabat-Zinn, J. (1990). Full catastrophe living: Using the wisdom of your body and mind to face stress, pain and illness. New York: Dell Publishing.

Kane, M.J., Bleckley, M.K., Conway, A.R.A., \& Engle, R.W. (2001). A controlled-attention view of working-memory capacity. Journal of Experimental Psychology: General, 130, 169-183.

Kane, M.J., \& Engle, R.W. (2003). Working-memory capacity and the control of attention: The contributions of goal neglect, response competition, and task set to Stroop interference. Journal of Experimental Psychology: General, 132, 47-70.

Keune, P.M., Bostanov, V., Hautzinger, M., \& Kotchoubey, B. (2013). Approaching dysphoric mood: State-effects of mindfulness meditation on frontal brain asymmetry. Biological Psychology, 93, 105-113.

Krygier, J.R., Heathers, J.A.J., Shahrestani, S., Abbott, M., Gross, J.J., \& Kemp, A.H. (2013). Mindfulness meditation, well-being, and heart-rate variability: A preliminary 
investigation into the impact of intensive Vipassana meditation. International Journal of Psychophysiology, 89, 305-313.

Lang, P.J., Bradley, M.M., \& Cuthbert, B.N. (2008). International affective picture system (IAPS): Affective ratings of pictures and instruction manual. Technical Report A-8. University of Florida, Gainesville, FL.

Lau, M.A., Bishop, S.R., Segal, Z.V., Buis, T., Anderson, N.D., Carlson, L., et al. (2006). The Toronto Mindfulness Scale: Development and validation. Journal of Clinical Psychology, $62,1445-1467$.

Levitt, J.T., Brown, T.A., Orsillo, S.M., \& Barlow, D.H. (2004). The effects of acceptance versus suppression of emotion on subjective and psychophysiological response to carbon dioxide challenge in patients with panic disorder. Behavior Therapy, 35, 747-766.

Lynch, T.R., Chapman, A.L., Rosenthal, M.Z., Kuo, J.R., \& Linehan, M.M. (2006). Mechanisms of change in Dialectical Behavior Therapy: Theoretical and empirical observations. Journal of Clinical Psychology, 62, 459-480.

Mankus, A.M., Aldao, A., Kerns, C., Mayville, E.W., \& Mennin, D.S. (2013). Mindfulness and heart rate variability in individuals with high and low generalized anxiety symptoms. Behaviour Research and Therapy, 51, 386-391.

McLaughlin, K.A., Mennin, D.S., \& Farach, F.J. (2007). The contributory role of worry in emotion generation and dysregulation generalized anxiety disorder. Behavior Research and Therapy, 45, 1735-1752.

Moscovitch, D.A., Suvak, M.K., \& Hofmann, S.G. (2010). Emotional response patterns during social threat in individuals with generalized social anxiety disorder and non-anxious controls. Journal of Anxiety Disorders, 24, 785-791. 
Moyer, C.A., Donnelly, M.P.W., Anderson, J.C., Valek, K.C., Huckaby, S.J., Wiederholt, D.A., Doty, R.L., Rehlinger, A.S., \& Rice, B.L. (2011). Frontal electroencephalographic asymmetry associated with positive emotion is produced by very brief meditation training. Psychological Science, 22, 1277-1279.

Olatunji, B.O., Forsyth, J.P., \& Feldner, M.T. (2007). Implications of emotion regulation for the shift from normative fear-relevant learning to anxiety-related psychopathology. American Psychologist, 62, 257-259.

Ortner, C.N.M., Kilner, S.J., \& Zelazo, P.D. (2007). Mindfulness meditation and reduced emotional interference on a cognitive task. Motivation and Emotion, 31, 271-283.

Ochsner, K.N., Bunge, S.A., Gross, J.J., \& Gabrieli, J.D. (2002). Rethinking feelings: An fMRI study of the cognitive regulation of emotion. Journal of Cognitive Neuroscience, 14, 1215-1229.

Papousek, I., Reiser, E.M., Weber, B., Freudenthaler, H.H., \& Schulter, G. (2012). Frontal brain asymmetry and affective flexibility in an emotional contagion paradigm. Psychophysiology, 49, 489-498.

Perlman, D.M., Salomons, T.V., Davidson, R.J., \& Lutz, A. (2010). Differential effects on pain intensity and unpleasantness of two meditation practices. Emotion, 10, 65-71.

Pond, R.S., Kashdan, T.B., DeWall, C.N., Savostyanova, A., Lambert, N.M., and Fincham, F.D. (2012). Emotion differentiation moderates aggressive tendencies in angry people: A daily diary analysis. Emotion, 12, 326-337.

Preacher, K.J., \& Hayes, A.F. (2008). Asymptotic and resampling strategies for assessing and comparing indirect effects in multiple mediator models. Behavior Research Methods, 40, 879-891. 
Richards, J.M., \& Gross, J.J. (2000). Emotion regulation and memory: The cognitive costs of keeping one's cool. Journal of Personality and Social Psychology, 79, 410-424.

Sobotka, S.S., Davidson, R.J., \& Senulis, J.A. (1992). Anterior brain electrical asymmetries in response to reward and punishment. Electroencephalography and Clinical Neurophysiology, 83, 236-247.

Schmeichel, B.J., Volokhov, R.N., \& Demaree, H.A. (2008). Working memory capacity and the self-regulation of emotional expression and experience. Journal of Personality and Social Psychology, 95, 1526-1540.

Sears, S., \& Kraus, S. (2009). I think therefore I ohm: Cognitive distortions and coping style as mediators of the effectiveness of mindfulness meditation on anxiety, positive and negative affect, and hope. Journal of Clinical Psychology, 65, 561-573.

Shapiro, S.L., Carlson, L.E., Astin, J.A., \& Friedman, B. (2006). Mechanisms of mindfulness. Journal of Clinical Psychology, 62, 373-386.

Shapiro, S.L., Brown, K.W., \& Biegel, G.M. (2007). Teaching self-care to caregivers: Effects of mindfulness-based stress reduction on the mental health of therapists in training. Training and Education in Professional Psychology, 1, 105-115.

Smith, T.W., Cribbet, M.R., Nealey-Moore, J.B., Uchino, B.N., Williams, P.G., MacKenzie, J., and Thayer, J.F. (2011). Matters of the variable heart: Respiratory sinus arrhythmia response to marital interaction and associations with marital quality. Journal of Personality and Social Psychology, 100, 103-119.

Stern, R.M., Ray, W.J., \&Quigley, K.S. (2001). Psychophysiological Recording. Oxford University Press: New York. 
Tang, Y., Ma, Y., Fan, Y., Feng, H., Wang, J., Feng, S.,...\& Fan, M. (2009). Central and autonomic nervous system interaction is altered by short-term meditation. Proceedings of the National Academy of Sciences of the United States of America, 106, 22, 8865-8870.

Teasdale, J.D., Segal, Z.V., Williams, J.M.G., Ridgeway, V.A., Soulsby, J.M., \& Lau, M.A. (2000). Prevention of relapse/recurrence in major depression by mindfulness-based cognitive therapy. Journal of Consulting and Clinical Psychology, 68, 615-623.

Thayer, J.F. \& Lane, R.D. (2009). Claude Bernard and the heart-brain connection: Further elaboration of a model of neurovisceral integration. Neuroscience and Biobehavioral Reviews, 33, 81-88.

Thayer, J.F., Ahs, F., Fredrikson, M., Sollers, J.J., III, \& Wager, T.D. (2012). A meta-analysis of heart rate variability and neuroimaging studies: implications for heart rate variability as a marker of stress and health. Neuroscience and Biobehavioral Reviews, 36, 747-756.

Tomarken, A.J., Davidson, R.J., \& Henriques, J.B. (1990). Resting frontal brain asymmetry predicts affective responses to films. Journal of Personality and Social Psychology, 59, 791-801.

van Vugt, M.K., \& Jha, A.P. (2011). Investigating the impact of mindfulness meditation training on working memory: A mathematical modeling approach. Cognitive Affective Behavioral Neuroscience, 11, 344-353.

Vernig, P.M., \& Orsillo, S.M. (2009). Psychophysiological and self-reported emotional responding in alcohol-dependent college students: The impact of brief mindfulness/acceptance instruction. Cognitive Nehaviour Therapy, 38, 174-183.

Unsworth, N., Heitz, R.P., Schrock, J.C., \& Engle, R.W. (2005). An automated version of the operation span task. Behavior Research Methods, 37, 498-505. 
Vujanovic, A.A., Bonn-Miller, M.O., Bernstein, A., McKee, L.G., \& Zvolensky, M.J. (2010). Incremental validity of mindfulness skills in relation to emotional dysregulation among a young adult community sample. Cognitive Behavior Therapy, 39, 203-213.

Watson, D., Clark, L.A., \& Tellegen, A. (1988). Development and validation of brief measures of positive and negative affect: The PANAS scales. Journal of Personality and Social Psychology, 54, 1063-1070.

Weber, B., Papousek, I., \& Schulter, G. (2011, July). Acoustical mood induction - development of new stimulus material (ECOS - emotionally contagious sound clips). Poster presented at the $12^{\text {th }}$ European Congress of Psychology, Istanbul, Turkey. 


\section{Appendix A}

International Affective Picture System Slides Used in Each Mood Induction Group

Negative

1019, 1051, 1090, 1111, 1200, 1205, 1274, 1301, 2095, 2120, 2141, 2661, 2688, 2691, $2700,2703,2710,2716,2717,2730,2745.2,2751,2799,2800,2900,2981,3015,3022,3190$, $3216,3220,3300,3350,4621,5961,6020,6200,6211,6213,6242,6243,6410,6555,6562$, 6571, 6825, 6838, 7359, 7361, 7380, 8230, 9005, 9006, 9007, 9040, 9042, 9120, 9160, 9180, 9270, 9301, 9340, 9373, 9400, 9424, 9425, 9426, 9427, 9430, 9433, 9470, 9490, 9495, 9592, 9611

Positive

$1340,1440,1463,1540,1640,1710,1720,1811,2058,2150,2160,2208,2209,2216$, 2303, 2340, 2345, 2352.1, 2605, 4150, 4180, 4220, 4250, 4310, 4520, 4532, 4533, 4542, 4598, 4599, 4609, 4611, 4614, 4617, 4623, 4624, 4626, 4640, 4641, 4653, 4680, 4700, 5260, 5270, 5450, 5460, 5480, 5600, 5623, 5660, 5833, 5910, 7220, 7230, 7260, 7289, 7330, 7400, 7460, 7501, 7502, 7508, 8021, 8090, 8210, 8340, 8350, 8380, 8420, 8496, 8500, 8501, 8502, 8503, 8531 


\title{
Appendix B
}

\author{
International Affective Digital Sounds Used in Each Mood Induction Group
}

Negative

$105,106,116,134,241,242,243,244,255,260,261,280,283,288,289,293,295,296$, $310,319,380,501,611,703,719$

Positive

$109,110,111,205,210,216,221,224,226,254,351,353,355,363,365,366,400,601$, $716,721,802,813,816,820,826$ 


\section{Appendix C}

Demographics Questionnaire

Please answer the following questions about yourself.

1. What is your age?

2. What is your gender? Male Female

3. Which of the following best describes your racial background?

African-American Caucasian Native American Hispanic
Asian

Other , describe:

4. Have you ever had a seizure? YES NO

If yes, at what age did you have your first seizure?

How many seizures have you had in total?

5. Have you ever had a stroke? YES $\mathrm{NO}$

If yes, at what age did you suffer from a stroke?

If yes, how many strokes have you suffered from?

6. Are you currently being treated or been advised to seek treatment for a brain tumor? YES NO

7. Have you ever suffered from any head injuries? (ex: falling, being in a vehicle accident, violence)? YES NO

If yes, please describe how you obtained this head injury

8. Do you currently take any prescription drugs?

If yes, please list the medications you take 


\section{Appendix D}

\section{Difficulties in Emotion Regulation Scale}

Please indicate how often the following statements apply to you by writing the appropriate number from the scale below on the line beside each item:

$\begin{array}{ccccc}\text { almost never } & \text { sometimes } & \text { about half the time } & \text { most of the time } & \text { almost always } \\ (0-10 \%) & (11-35 \%) & (36-65 \%) & (66-90 \%) & (91-100 \%)\end{array}$

\footnotetext{
1) I am clear about my feelings.
}

2) I pay attention to how I feel.

3) I experience my emotions as overwhelming and out of control.

4) I have no idea how I am feeling.

5) I have difficulty making sense out of my feelings.

6) I am attentive to my feelings.

7) I know exactly how I am feeling.

8) I care about what I am feeling.

9) I am confused about how I feel.

10) When I'm upset, I acknowledge my emotions.

11) When I'm upset, I become angry with myself for feeling that way.

12) When I'm upset, I become embarrassed for feeling that way.

13) When I'm upset, I have difficulty getting work done.

14) When I'm upset, I become out of control.

15) When I'm upset, I believe that I will remain that way for a long time.

16) When I'm upset, I believe that I'll end up feeling very depressed.

17) When I'm upset, I believe that my feelings are valid and important.

18) When I'm upset, I have difficulty focusing on other things.

19) When I'm upset, I feel out of control.

20) When I'm upset, I can still get things done.

21) When I'm upset, I feel ashamed with myself for feeling that way.

22) When I'm upset, I know that I can find a way to eventually feel better. 
23) When I'm upset, I feel like I am weak.

24) When I'm upset, I feel like I can remain in control of my behaviors.

25) When I'm upset, I feel guilty for feeling that way.

26) When I'm upset, I have difficulty concentrating.

27) When I'm upset, I have difficulty controlling my behaviors.

28) When I'm upset, I believe that there is nothing I can do to make myself feel better.

29) When I'm upset, I become irritated with myself for feeling that way.

30) When I'm upset, I start to feel very bad about myself.

31) When I'm upset, I believe that wallowing in it is all I can do.

32) When I'm upset, I lose control over my behaviors.

33) When I'm upset, I have difficulty thinking about anything else.

34) When I'm upset, I take time to figure out what I'm really feeling.

35) When I'm upset, it takes me a long time to feel better.

36) When I'm upset, my emotions feel overwhelming. 


\author{
Appendix E \\ Mindful Attention Awareness Scale
}

\title{
Day-to-Day Experiences
}

Instructions: Below is a collection of statements about your everyday experience. Using the 1-6 scale below, please indicate how frequently or infrequently you currently have each experience. Please answer according to what really reflects your experience rather than what you think your experience should be. Please treat each item separately from every other item.

1 - Almost Always 2- Very Frequently 3-Somewhat Frequently 4- Somewhat Infrequently 5- Very Infrequently 6- Almost Never

1. I could be experiencing some emotion and not be conscious of it until some time later.

2. I break or spill things because of carelessness, not paying attention, or thinking of something else.

3. I find it difficult to stay focused on what's happening in the present.

4. I tend to walk quickly to get where I'm going without paying attention to what I experience along the way.

5. I tend not to notice feelings of physical tension or discomfort until they really grab my attention.

6. I forget a person's name almost as soon as I've been told it for the first time.

7. It seems I am "running on automatic," without much awareness of what I'm doing.

8. I rush through activities without being really attentive to them.

9. I get so focused on the goal I want to achieve that I lose touch with what I'm doing right now to get there.

10. I do jobs or tasks automatically, without being aware of what I'm doing.

11. I find myself listening to someone with one ear, doing something else at the same time.

12. I drive places on 'automatic pilot' and then wonder why I went there.

13. I find myself preoccupied with the future or the past.

14. I find myself doing things without paying attention.

15. I snack without being aware that I'm eating. 


\section{Appendix F}

The Positive and Negative Affect Scale

This scale consists of a number of words that describe different feelings and emotions. Read each item and then mark the appropriate answer in the space next to that word. Indicate to what extent you feel this way right now, that is, at the present moment. Use the following scale to record your answers.

1- very slightly or not at all 2- a little 3-moderately 4- quite a bit 5- extremely

_ interested

_ distressed

_ excited

- upset

_ strong

_ guilty

- scared

_ hostile

_ enthusiastic

_ proud

_ irritable

_ alert

_ ashamed

_ inspired

_ nervous

_ determined

_ attentive

_ jittery

_ active

_ afraid 


\section{Appendix G}

State Difficulties in Emotion Regulation Scale

Please indicate how the following statements apply to you IN THIS PRESENT MOMENT by writing the appropriate number from the scale below on the line beside each item:

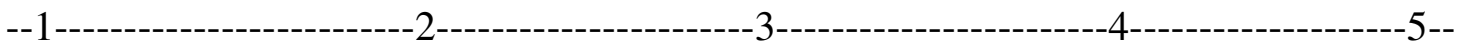

Not at All

Completely

$(0-10 \%)$

$(11-35 \%)$

$(36-65 \%)$

$(66-90 \%)$

$(91-100 \%)$

1) I am clear about my feelings.

2) I pay attention to how I feel.

3) I experience my emotions as overwhelming and out of control.

4) I have no idea how I am feeling.

5) I have difficulty making sense out of my feelings.

6) I am attentive to my feelings.

7) I know exactly how I am feeling.

8) I care about what I am feeling.

9) I am confused about how I feel.

10) I acknowledge my emotions.

11) I become angry with myself for feeling this way.

12) I become embarrassed for feeling this way.

13) I have difficulty getting work done.

14) I become out of control.

15) I believe that I will remain this way for a long time.

16) I believe that I'll end up feeling very depressed.

17) I believe that my feelings are valid and important.

18) I have difficulty focusing on other things.

19) I feel out of control.

20) I can still get things done.

21) I feel ashamed with myself for feeling this way.

22) I know that I can find a way to eventually feel better. 
23) I feel like I am weak.

24) I feel like I can remain in control of my behaviors.

25) I feel guilty for feeling this way.

26) I have difficulty concentrating.

27) I have difficulty controlling my behaviors.

28) I believe that there is nothing I can do to make myself feel better.

29) I become irritated with myself for feeling this way.

30) I start to feel very bad about myself.

31) I believe that wallowing in it is all I can do.

32) I lose control over my behaviors.

33) I have difficulty thinking about anything else.

34) I take time to figure out what I'm really feeling.

35) It takes me a long time to feel better.

36) My emotions feel overwhelming. 


\section{Appendix $\mathrm{H}$}

\section{The Toronto Mindfulness Scale}

Instructions: We are interested in what you just experienced. Below is a list of things that people sometimes experience. Please read each statement. Next to each statement are five choices: "not at all," "a little," "moderately," "quite a bit," and "very much." Please indicate the extent to which you agree with each statement. In other words, how well does the statement describe what you just experienced, just now?

1. I experienced myself as separate from my changing thoughts and feelings.

2. I was more concerned with being open to my experiences than controlling or changing them.

3. I was curious about what I might learn about myself by taking notice of how I react to certain thoughts, feelings or sensations.

4. I experienced my thoughts more as events in my mind than as a necessarily accurate reflection of the way things 'really' are.

5. I was curious to see what my mind was up to from moment to moment.

6. I was curious about each of the thoughts and feelings that I was having.

7. I was receptive to observing unpleasant thoughts and feelings without interfering with them.

8. I was more invested in just watching my experiences as they arose, than in figuring out what they could mean.

9. I approached each experience by trying to accept it, no matter whether it was pleasant or unpleasant.

10. I remained curious about the nature of each experience as it arose.

11. I was aware of my thoughts and feelings without overidentifying with them.

12. I was curious about my reactions to things.

13. I was curious about what I might learn about myself by just taking notice of what my attention gets drawn to. 


\section{Appendix I}

\section{Mindfulness Condition Instructions}

For the next several minutes, I'm going to ask you to think about, and try, a particular kind of awareness, called mindfulness. The term mindfulness comes from Eastern spiritual and religious traditions, but psychology has begun to find that mindfulness (without the spiritual and religious context) can be helpful for people in many ways. Today I'm just going to tell you a little bit about this way of paying attention, and have you try it out, to see what it's like for you. Mindfulness is paying attention in the present moment, with openness and curiosity, instead of judgment. We often focus on things other than what is happening in the moment-worrying about the future, thinking about the past, focusing on what is coming next rather than what is right in front of us. And it is useful that we can do a number of things without paying attention to them. However, sometimes it is helpful to bring our attention, particularly a curious and kind attention, to what we are doing in the moment. Sometimes we do pay close attention to what we are thinking and feeling and we become very critical of our thoughts and feelings and we try to either change them or distract ourselves because this critical awareness can be very painful. For example, we might notice while we are talking to someone new that our voice is shaky, or we aren't speaking clearly, and think, "I'm such an idiot! What is wrong with me? If I don't calm down, this person will never like me!"

Being mindful falls between these two extremes-we pay attention to what is happening inside and around us, we see events and experiences as what they are, and we allow things we can't control to be as they are while we focus our attention on the task at hand. For example, when talking to someone new we might notice those same changes in our voice, take a moment to reflect, "This is how it is now, there go my thoughts again," and gently bring our attention back to the person and our conversation. This second part of mindfulness, holding our judgments loosely and not trying to change our thoughts or feelings can be especially hard. In fact, often being mindful involves practicing not judging our tendency to have judgments! Mindfulness is a process: We do not reach a final and total state of mindfulness. It is a way of being in one moment that comes and goes. Mindfulness is losing our focus 100 times and returning to it 101 times. The best way to understand mindfulness is to practice it, so let's do that now. 


\section{Mindfulness Exercise 1}

First, just allow your eyes to close gently, or to lower . . . and bring yourself to sit in an upright position ... begin by noticing how you are sitting in the chair . . noticing the places where you are touching the chair, the places where you are touching the floor . . noticing where the air is touching your skin and what that feels like ... notice any sounds in the room... and now gently drawing your attention to your breath ... noticing (without trying to change it) where your breath is coming from . . . noticing where it enters your body when you inhale ... how it travels through your body before you exhale it . . . Noticing how your body moves with each inhalation, each exhalation ... allowing any thoughts or feelings that occur to naturally rise and fall, without trying to hold onto them or get rid of them . . . just continue bringing your awareness to your experience in this moment ... and continuing to notice your breath ... take a few moments now as you allow whatever comes to come and whatever goes to go and whatever stays to stay ... if your mind wanders, that is okay, just gently bring your attention back to this moment... and again bringing your awareness to the room...to the way you are sitting in the chair...take one more deep breath and gradually open your eyes.

One of the hardest times to be mindful is when we are experiencing a strong emotion, like fear, or sadness, or joy. In those moments, we often want to either hold on to the emotion or get rid of it, rather than allowing it to rise and fall naturally. And sometimes it feels like we can make emotions stay or make them leave, but other times we may find that trying to make an emotion stay makes it leave even faster, while trying to get rid of it keeps it hanging around. Also, emotions can give us important information about our lives, a particular situation, or the way someone we care about is responding to us. So it can be useful for us to notice the emotions we are having as they happen, rather than judging them or trying to change them. We can bring the same kind of awareness you just practiced to any emotional experience, noticing what we feel in our bodies, what thoughts we have, and just letting that experience happen without getting caught up in it. Our feelings will change on their own when we let them be, rather than seeing them as bad or good or something to be changed. This is also something that is easier to experience than it is to describe. Let's do another exercise to give you a sense of what I'm describing. 


\section{Mindfulness Exercise 2: Mindfulness of Emotions}

First, make yourself comfortable in your chair. Take a few moments to notice your breathing.

Close your eyes, and focus on your breath . . . Noticing how breath travels into your body, through your body, and back out of your body ... noticing any tension in your body ... and gently letting it go .... Spending a few moments just focusing your attention on your breath ... Now I would like to read a poem to you as your eyes remain closed and have you notice any reactions to the poem that arise.

The Guest House by Rumi (1995)

This being human is a guest house.

Every morning a new arrival.

A joy, a depression, a meanness, some momentary awareness comes as an unexpected visitor.

Welcome and entertain them all!

Even if they are a crowd of sorrows, who violently sweep your house empty of all its furniture, still, treat each guest honorably. He may be clearing you out for some new delight.

The dark thought, the shame, the malice, meet them at the door laughing and invite them in. Be grateful for whatever comes, because each has been sent as a guide from beyond.

...now with your eyes still closed gently draw your attention back to your breath . . noticing how your body moves with each inhalation, each exhalation . . allowing any thoughts or feelings that occur to naturally rise and fall, without trying to hold onto them or get rid of them . . . just 
continue bringing your awareness to your experience in this moment . . . and continue to notice your breath . . . take a few moments as you allow whatever comes to come and whatever goes to go and whatever stays to stay ... if your mind wanders, that is okay, just gently bring your attention back to this moment... just continue bringing your awareness to your experience in this moment ... and continue to notice your breath... and again bringing your awareness to the room... to the way you are sitting in the chair...take one more deep breath...and gradually open your eyes 


\author{
Appendix J \\ Consent Form
}

Study Title: Mindfulness, Emotion Regulation, and Psychological Well-being: A Mediational Relationship?

Investigators: Tanya S. Nichols, B.A. and Jane Stafford, Ph.D.

Introduction: You are being asked to participate in a research study. You should read this form carefully and feel free to ask the investigators any questions that you may have before making a decision whether or not to participate. The research is being conducted by Tanya Nichols, a graduate student in the Department of Psychology at the University of South Carolina Aiken, under the supervision of Dr. Jane Stafford. Please note that you must be at least 18 years of age or older to participate in this research.

Purpose of Study: Mindfulness can be described as a mental state that includes nonjudgmental awareness of and attention to experiences as they unfold in the present moment. Research has demonstrated that cultivating various aspects of the mind through the use of mindful attention has resulted in increased well-being. The goal of the study is to assess whether mindfulness and emotional experience are related to memory.

Study Procedures: You will be asked to respond to questions (e.g., "How are you feeling right now?") regarding traits you may or may not have as well as emotions you may or may not be experiencing. Also, your heart rate and brain activity will be measured by attaching sensors to the palm of your hand, your ankles, and your forehead. You will be asked to listen to information about mindfulness or an excerpt from National Public Radio. Next, you will be asked to experience a presentation of positive or negative pictures (e.g., a gun pointed at a person) and sounds (e.g., a baby crying) for about 10 minutes. Finally, you will be asked to complete a computer task that requires you to solve mathematical problems while performing a short-term memory test. The duration of the study is 1.5 hours.

Benefits of Participation: If you complete the entire study, you will receive 1.5 hours of experimental participation credit. Furthermore, this research may help us understand the relationship between mindfulness, emotions and working memory.

Risks of Participation: Some people may experience distress related to the emotional nature of the pictures and sounds as well as the experience of examining their own feelings and thoughts through answering questions. In case you feel any discomfort after completing this task and would like someone to talk to, you may contact the Counseling Center on the University of South Carolina at Aiken's campus at (803) 641-3609 or stop by Room 126 in the Business and Education Building.

Confidentiality: All of your information will be kept as confidential as possible. All information will be identified by a unique code number, and only the research investigators will have access to the information. Study information will be stored in locked filing cabinets and in password protected computer files at the University of South Carolina Aiken. The results of the study may be published or presented at meetings, but your identity will not be revealed. Records that identify you and the consent form signed by you may be inspected by the University's Institutional Review Board.

Voluntary Participation: Participation in the study is voluntary. Therefore, you may withdraw from the study at any time or decide not to answer any question you are not comfortable answering. If you begin the study and later decide to withdraw, you will be given credit for the amount of time you spent.

Contact Person: If you have any questions or problems regarding the study, you may contact me at tsnichols@usca.edu or my faculty advisor at jstafford@usca.edu. If you have any questions about your rights as a research participant, you may contact the Office of Research Compliance at the University of South Carolina at 803-777-7095. 
Signature/Date: I have read (or have had read to me) the contents of this consent form and have been encouraged to ask questions. If I had questions, I received answers to them. I give my consent to participate in this study. I have received (or will receive) a copy of this form for my records and future reference.

Participant's Signature/Date

Investigator's Signature/Date 
Table 1

TMS scores at Time 1 (immediately following the intervention) and Time 2 (completion of experimental session) for the Mindfulness and Control Groups

\begin{tabular}{lcccc}
\hline & \multicolumn{2}{c}{ Mindfulness Group } & \multicolumn{2}{c}{ Control Group } \\
\hline & Mean & $S D$ & Mean & $S D$ \\
\hline Time 1: & & & 38.78 & 9.12 \\
TMS Total & 43.34 & 8.77 & 18.16 & 6.07 \\
Curiosity subscale & 20.13 & 5.72 & 20.63 & 4.50 \\
Decentering subscale & 23.21 & 4.77 & & \\
Time 2: & & & 39.47 & 10.18 \\
TMS Total & 40.37 & 9.92 & 18.50 & 7.24 \\
Curiosity subscale & 18.76 & 6.12 & 20.97 & 4.83 \\
Decentering subscale & 21.61 & 5.12 & & \\
\hline
\end{tabular}


Table 2

PANAS positive and negative scores and DERS-S scores for the Mindfulness and Control Groups

Mindfulness Group Control Group

\begin{tabular}{lcccccccc}
\hline & \multicolumn{2}{c}{ Positive Induction } & \multicolumn{2}{c}{ Negative Induction } & \multicolumn{2}{c}{ Positive Induction } & \multicolumn{2}{c}{ Negative Induction } \\
\hline & Mean & $S D$ & Mean & $S D$ & Mean & $S D$ & Mean & $S D$ \\
\hline PANAS positive & 33.10 & 7.58 & 26.41 & 7.95 & 26.57 & 9.38 & 24.39 & 7.76 \\
PANAS negative & 13.19 & 3.86 & 19.47 & 7.14 & 15.71 & 2.56 & 16.44 & 5.78 \\
DERS-S & 7.48 & 4.42 & 10.88 & 5.95 & 8.07 & 2.17 & 9.44 & 4.08 \\
Nonacceptance & & & & & & & & \\
DERS-S Goals & 9.38 & 4.31 & 11.65 & 3.73 & 10.64 & 3.63 & 8.72 & 3.75 \\
DERS-S Impulse & 7.62 & 2.94 & 9.88 & 4.06 & 8.57 & 2.53 & 7.94 & 2.62 \\
DERS-S & 11.95 & 4.80 & 13.35 & 5.12 & 12.86 & 4.56 & 13.11 & 5.82 \\
Awareness & & & & & & & & 4.48 \\
DERS-S & 11.38 & 3.32 & 14.53 & 5.87 & 13.00 & 4.37 & 11.83 \\
Strategies & & & & & & & & 4.63 \\
DERS-S Clarity & 9.05 & 4.13 & 10.12 & 3.42 & 8.86 & 2.98 & 9.50 & 4.66 \\
DERS-S Total & 56.86 & 17.61 & 70.41 & 21.03 & 62.00 & 12.97 & 60.56 & 17.48 \\
\hline
\end{tabular}




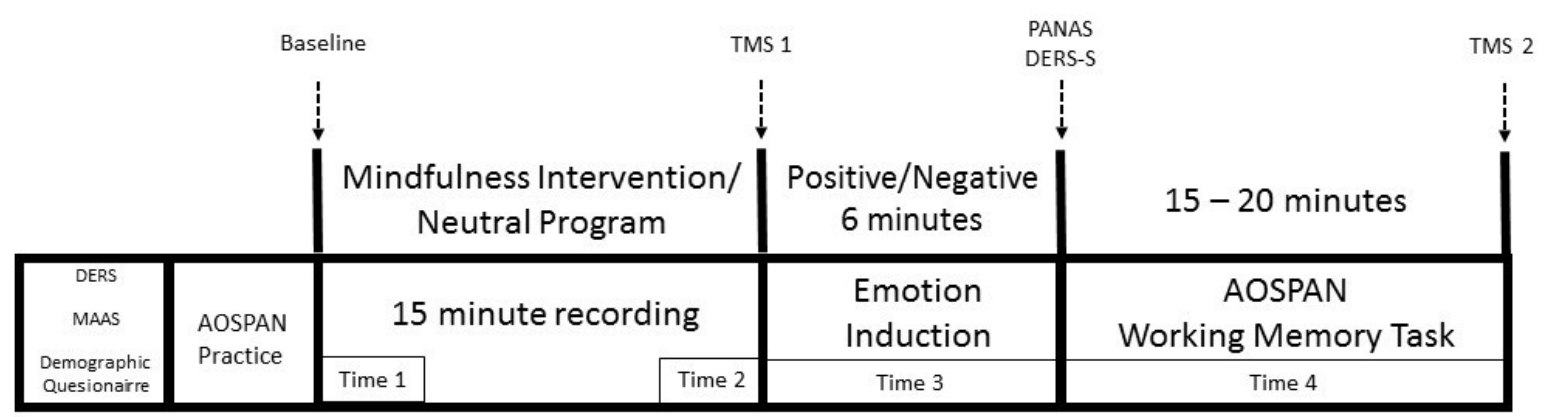

Figure 1. Schematic diagram of the procedure. DERS = Difficulties in Emotion Regulation Scale; MAAS = Mindfulness Awareness and Attention Scale; PANAS = Positive and Negative Affect Schedule; DERS-S = Difficulties in Emotion Regulation Scale - State; TMS = Toronto Mindfulness Scale; Time 1, 2, 3 and 4 = times at which physiological data used for analyses. 


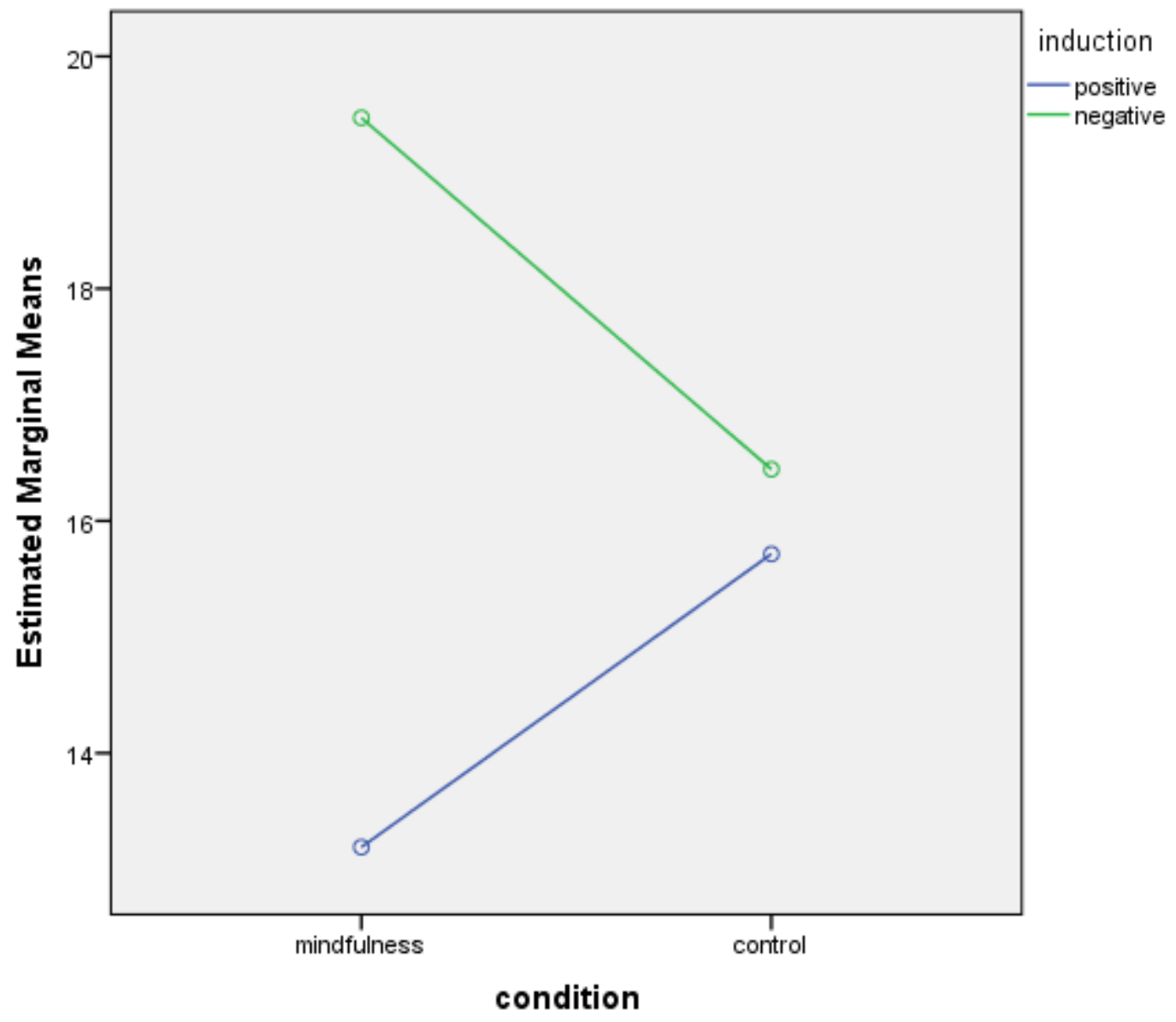

Figure 2. Graph of the interaction of Condition (Mindfulness vs. Control) and Induction (Positive vs. Negative) regarding Negative Affect 


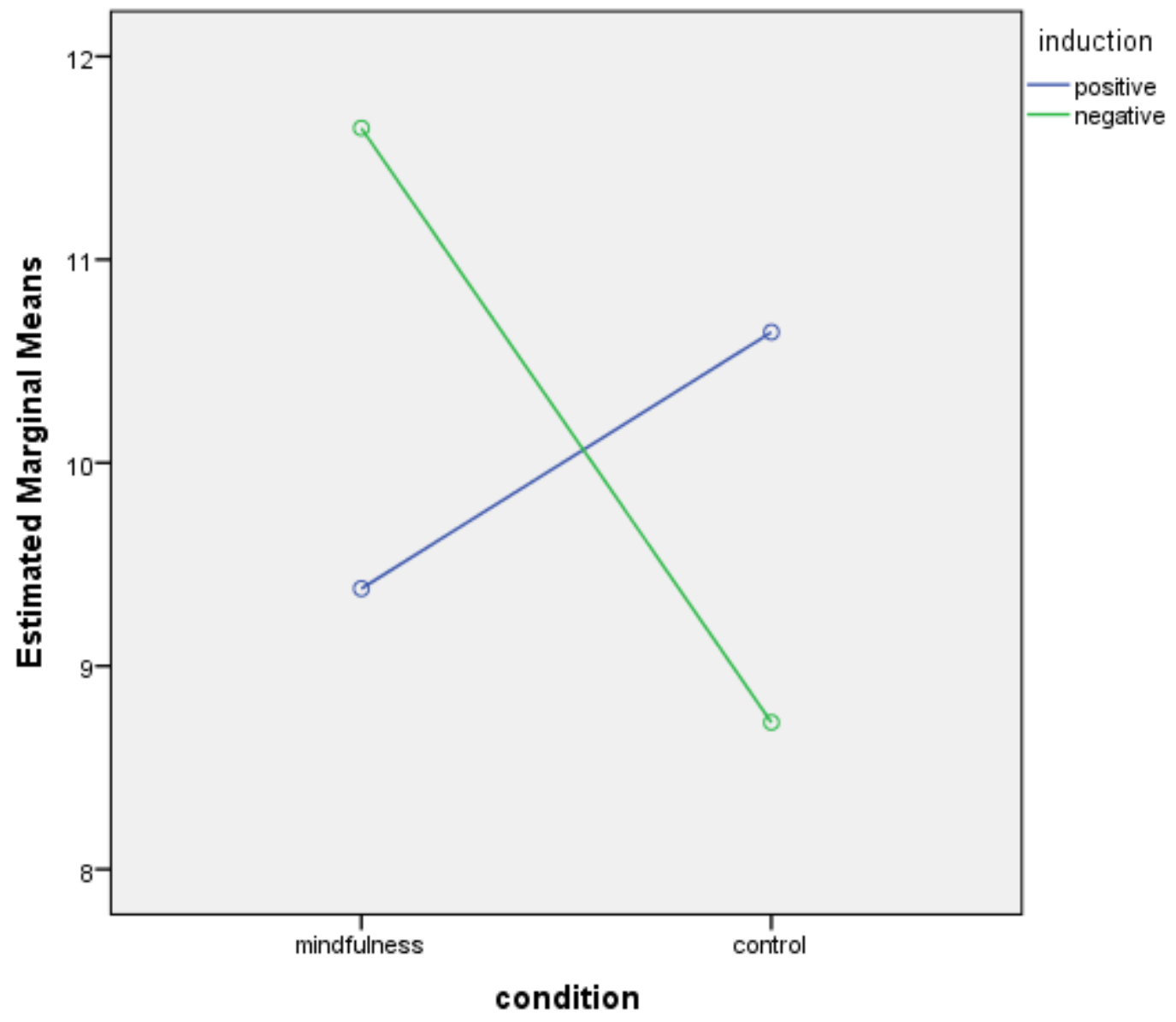

Figure 3. Graph of the interaction of Condition (Mindfulness vs. Control) and Induction (Positive vs. Negative) regarding Goal-Directed Behavior (GOALS) 


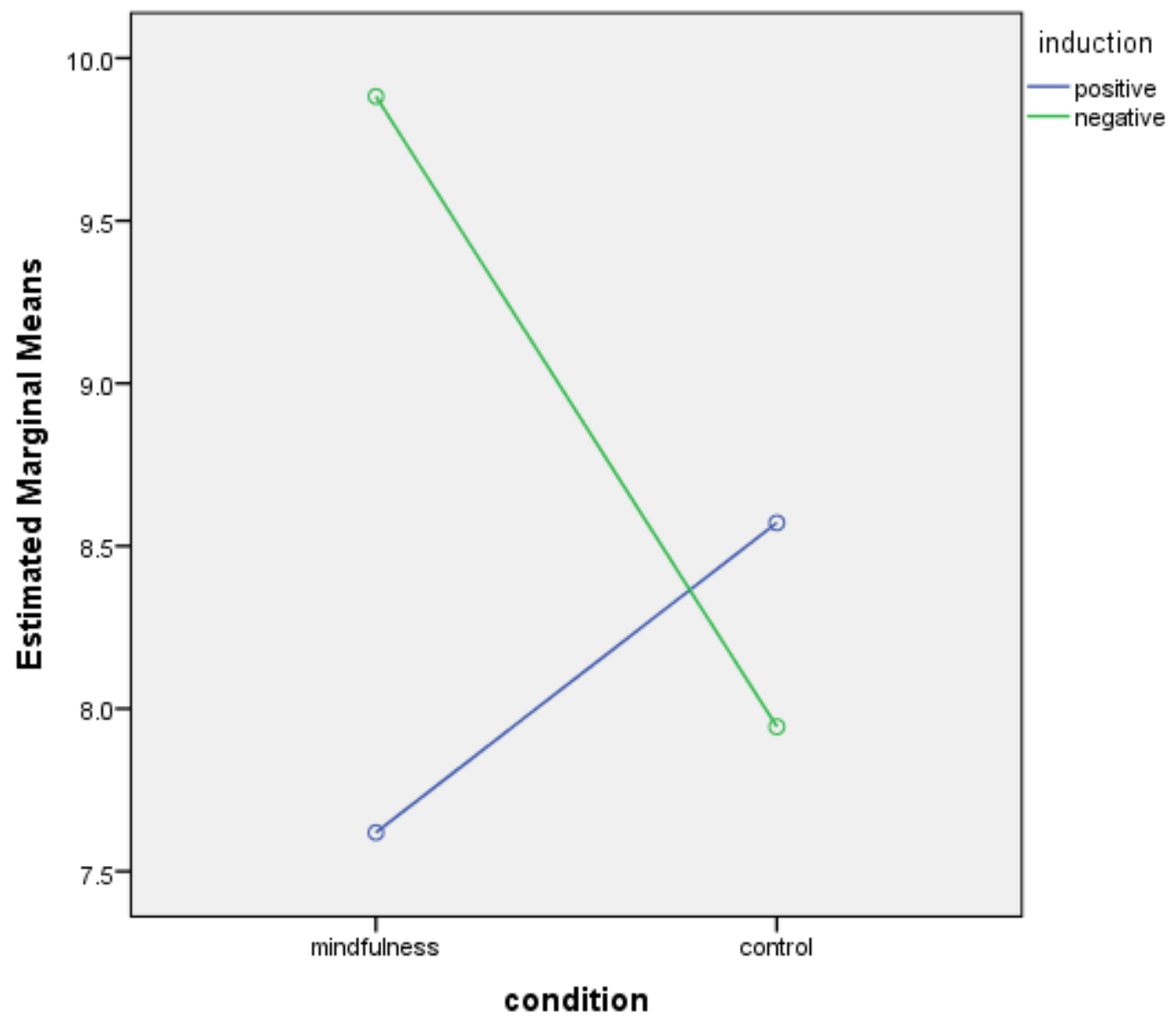

Figure 4. Graph of the interaction of Condition (Mindfulness vs. Control) and Induction (Positive vs. Negative) regarding Impulse Control Difficulties (IMPULSE) 


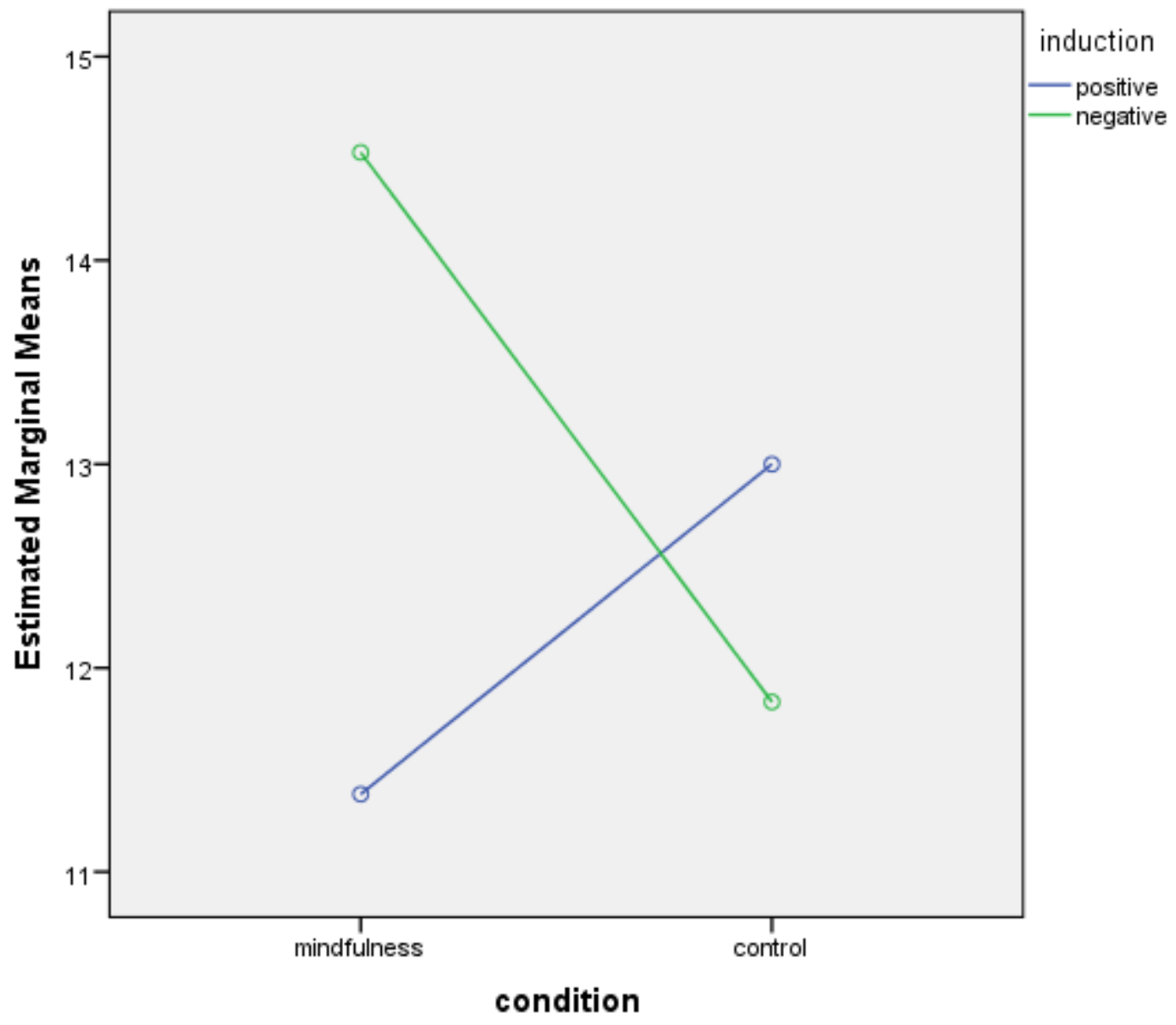

Figure 5. Graph of the interaction of Condition (Mindfulness vs. Control) and Induction (Positive vs. Negative) regarding Limited Access to Emotion Regulation Strategies (STRATEGIES) 

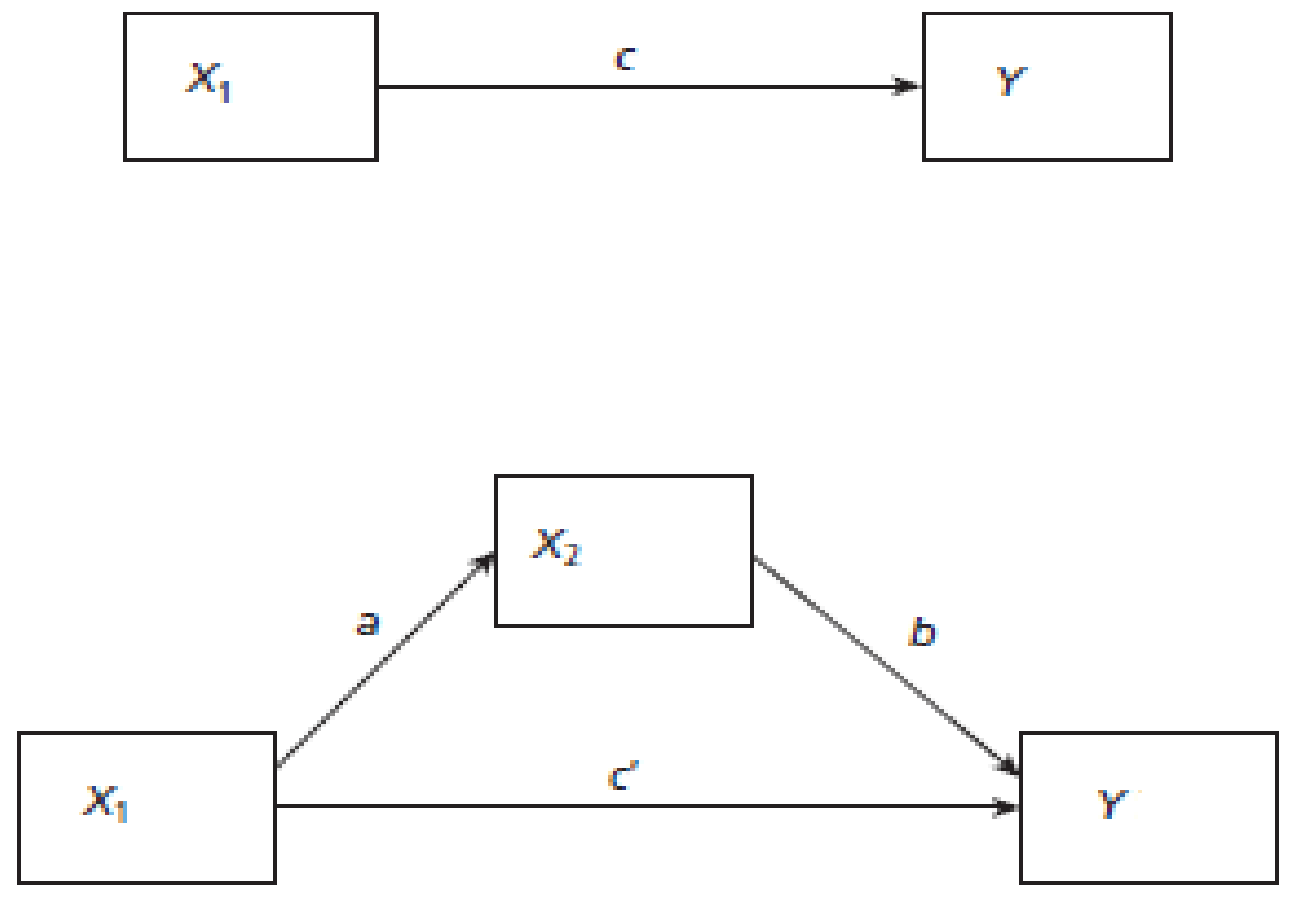

Figure 6. Hypothetical mediation example. $\mathrm{X}_{1}=$ independent variable (IV); $\mathrm{X}_{2}=$ mediational variable $(M) ; \mathrm{Y}=$ dependent variable $(\mathrm{DV}) . c=$ total effect of $\mathrm{X}_{1}$ (including the effect of the mediator: $\mathrm{X}_{2}$ ) on $\mathrm{Y} ; a=$ direct effect of $\mathrm{X}_{1}$ on $\mathrm{X}_{2} ; b=$ direct effect of $\mathrm{X}_{2}$ on $\mathrm{Y} ; c^{\prime}=$ the direct effect of $\mathrm{X}_{1}$ (not mediated by $\mathrm{X}_{2}$ ) on $\mathrm{Y}$. 